\title{
Domination chain: Characterisation, classical complexity, parameterised complexity and approximability
}

\author{
Cristina Bazgan ${ }^{\mathrm{a}, 1}$, Ljiljana Brankovic $^{\mathrm{b}}$, Katrin Casel $^{\mathrm{c}}$, Henning Fernau ${ }^{\mathrm{c}, *}$ \\ a Université Paris-Dauphine, PSL Research University, CNRS, UMR 7243, LAMSADE, 75016 Paris, France \\ b School of Electrical Engineering and Computer Science, The University of Newcastle, Callaghan, NSW 2308, Australia \\ ' Theoretische Informatik, Abteilung Informatikwissenschaften, Fachbereich 4, Universität Trier, D-54286 Trier, Germany
}

\section{A R T I C L E I N F O}

\section{Article history:}

Received 7 June 2016

Received in revised form 27 August 2019

Accepted 1 October 2019

Available online 14 October 2019

\section{Keywords:}

Domination chain

Classical complexity

Parameterised complexity

Approximation complexity

Special graph classes

\begin{abstract}
A B S T R A C T
We survey the most important results regarding the domination chain parameters, including the characterisation of the domination sequence, complexity of exact and parameterised algorithms, and approximation and inapproximability ratios. We provide a number of new results for the upper and lower irredundance and their complements, and a few results for other domination chain problems. In particular, we analyse the structure of maximum irredundant sets and we provide new bounds on the upper irredundance number. We show several approximability results for upper and lower irredundance and their complements on general graphs; all four problems remain NP-hard even on planar cubic graphs and APX-hard on cubic graphs. Finally, we give some results on everywhere dense graphs, and study some related extension problems.
\end{abstract}

(C) 2019 Elsevier B.V. All rights reserved.

\section{Introduction}

In this paper, we present a comparative study on domination, independence and irredundance in graphs. We focus both on combinatorial and computational aspects of the domination chain and provide several new results regarding combinatorial bounds on the domination parameters, as well as the approximability and parameterised complexity of the corresponding problems.

Throughout the paper, we use $G=(V, E)$ to denote an undirected graph with the set of vertices $V$ and the set of edges $E$. For any subset of vertices $V^{\prime} \subseteq V$ we define a subgraph $G\left[V^{\prime}\right]$ of $G$ induced by $V^{\prime}$ as a graph $G\left[V^{\prime}\right]=\left(V^{\prime}, E^{\prime}\right)$ such that for any pair of vertices $u, v$ with $v \in V^{\prime}$ and $u \in V^{\prime}$, there is an edge $(u, v) \in E^{\prime}$ if and only if $(u, v) \in E$. We use $N(v)$ and $N[v]$ to denote the open and closed neighbourhood of the vertex $v \in V$, respectively; formally, $N(v)=\{u \in V:(u, v) \in E\}$ and $N[v]=N(v) \cup\{v\}$. Similarly, we use $N\left(V_{1}\right)$ and $N\left[V_{1}\right]$ to denote the open and closed neighbourhood of the subset of vertices $V_{1} \subseteq V$, respectively, where $N\left(V_{1}\right)=\bigcup_{v \in V_{1}} N(v)$ and $N\left[V_{1}\right]=\bigcup_{v \in V_{1}} N[v]$.

We define the dominating, independent and irredundant sets as follows.

An independent set is a subset $I \subseteq V$ of vertices of $G=(V, E)$ such that $G[I]$ has no edges, that is, $G[I]=(I, \varnothing)$.

\footnotetext{
* Corresponding author.

E-mail addresses: bazgan@lamsade.dauphine.fr (C. Bazgan), ljiljana.brankovic@newcastle.edu.au (L. Brankovic), Casel@uni-trier.de (K. Casel), Fernau@uni-trier.de (H. Fernau).

1 Institut Universitaire de France.
} 
Table 1

The six parameters in the domination chain.

\begin{tabular}{|c|c|c|}
\hline Parameter & Name & Description \\
\hline $\operatorname{ir}(G)$ & Lower irredundance number & $\begin{array}{l}\text { The size of the minimum maximal } \\
\text { irredundant set in a graph } G \text {. }\end{array}$ \\
\hline$\gamma(G)$ & (Lower) Domination number & $\begin{array}{l}\text { The size of the minimum } \\
\text { dominating set in a graph } G \text {. }\end{array}$ \\
\hline$i(G)$ & Lower independence number & $\begin{array}{l}\text { The size of the minimum maximal } \\
\text { independent set in a graph } G \text {. }\end{array}$ \\
\hline$\alpha(G)$ & (Upper) Independence number & $\begin{array}{l}\text { The size of the maximum } \\
\text { independent set in a graph } G \text {. }\end{array}$ \\
\hline$\Gamma(G)$ & Upper domination number & $\begin{array}{l}\text { The size of the maximum minimal } \\
\text { dominating set in a graph } G \text {. }\end{array}$ \\
\hline $\operatorname{IR}(G)$ & Upper irredundance number & $\begin{array}{l}\text { The size of the maximum } \\
\text { irredundant set in a graph } G \text {. }\end{array}$ \\
\hline
\end{tabular}

A dominating set is a subset $D \subseteq V$ of vertices of $G=(V, E)$ such that every vertex in $V$ is either in $D$ or has a neighbour in $D$; formally, $D \subseteq V$ is a dominating set if $N[D]=V$.

An irredundant set is a subset $S \subseteq V$ of vertices in $G=(V, E)$ such that for all $s \in S, N[s]-N[S-\{s\}] \neq \emptyset$.

In 1978, Cockayne, Hedetniemi and Miller [33] introduced the well-known domination chain that links parameters related to the fundamental notions of independence, domination and irredundance in graphs. The domination chain was further studied by Bollobas and Cockayne [21], and later by Haynes, Hedetniemi and Slater [53] and many others $[19,32,62,68]$.

In the domination chain, $\alpha(G)$ and $i(G)$ are used to denote the maximum and minimum cardinalities over all maximal independent sets in $G, \gamma(G)$ and $\Gamma(G)$ the minimum and maximum cardinalities over all minimal dominating sets in $G$, and $\operatorname{IR}(G)$ and $\operatorname{ir}(G)$ the maximum and the minimum cardinality over all maximal irredundant sets in $G$. For every graph $G$ these parameters satisfy

$$
\operatorname{ir}(G) \leq \gamma(G) \leq i(G) \leq \alpha(G) \leq \Gamma(G) \leq \operatorname{IR}(G) .
$$

For the ease of reference, the six parameters of the domination chain are defined in Table 1. Over time, other parameters were added to the domination chain (see, for example, [51]).

An interesting question that one can ask is whether the parameters of the domination chain can be arbitrary positive integers satisfying only the above inequalities. To answer this question we first define a 'domination sequence'.

A sequence of positive integers $a_{1}, a_{2}, a_{3}, a_{4}, a_{5}, a_{6}$ is called a domination sequence if and only if there exists a graph $G$ such that $\operatorname{ir}(G)=a_{1}, \gamma(G)=a_{2}, i(G)=a_{3}, \alpha(G)=a_{4}, \Gamma(G)=a_{5}$ and $\operatorname{IR}(G)=a_{6}$. In 1993, Cockayne and Mynhardt [34] gave a necessary and sufficient condition for a sequence to be a domination sequence as stated in the following theorem.

Theorem 1 ([34]). Positive integers $a_{1} \leq a_{2} \leq a_{3} \leq a_{4} \leq a_{5} \leq a_{6}$ form a domination sequence if and only if

1. $a_{2} \leq 2 a_{1}-1$,

2. if $a_{1}=1$ then $a_{2}=a_{3}=1$, and

3. if $a_{4}=1$ then $a_{1}=a_{2}=a_{3}=a_{4}=a_{5}=a_{6}=1$.

In 2005, Zverovich and Zverovich [74] obtained a somewhat weaker result for cubic graphs.

Theorem 2 ([74]). For any non-negative integers $k_{1}, k_{2}, k_{3}, k_{4}$ and $k_{5}$, there exists a cubic graph $G$ satisfying the following conditions: $\gamma(G)-\operatorname{ir}(G) \geq k_{1}, i(G)-\gamma(G) \geq k_{2}, \alpha(G)-i(G) \geq k_{3}, \Gamma(G)-\alpha(G) \geq k_{4}$ and $\operatorname{IR}(G)-\Gamma(G) \geq k_{5}$.

In the next subsection, we give the notations and define the problems related to the parameters featuring in the original domination chain in Eq. (1).

\subsection{Definitions}

Throughout this paper, we use standard terminology from graph theory. Given a graph $G=(V, E)$, we use $n(G)$ to denote the order of $G$, that is, $n(G)=|V|$. We use $d(v)$ to denote the degree of a vertex $v$. We use $\Delta(G)$ and $\delta(G)$ to denote the maximum and the minimum degree in $G$, respectively, and for simplicity we often write just $\Delta$ and $\delta$ when $G$ is clear from the context.

A graph $G$ is planar if there exists an embedding of $G$ in the plane such that no two edges cross in a point other than an end vertex of one of the edges. A graph $G$ is outerplanar, or 1-outerplanar, if there exists an embedding of $G$ in the plane such that all the vertices of $G$ belong to the outer face. A graph $G$ is $k$-outerplanar if it has a planar embedding 
Table 2

The six problems in the domination chain. The input is the same for all the problems: a graph $G(V, E)$ and a non-negative integer $k$. For each problem, the alternative name used in this paper is given in bold.

\begin{tabular}{|c|c|c|}
\hline Parameter & $\begin{array}{l}\text { Problem } \\
\text { (Alternative names) }\end{array}$ & Question \\
\hline $\operatorname{ir}(G)$ & $\begin{array}{l}\text { LOWER IRREDUNDANCE } \\
\text { MINIMUM MAXIMAL IRREDUNDANT SET }\end{array}$ & $\begin{array}{l}\text { Does } G \text { have a maximal } \\
\text { irredundant set } S \\
\text { such that }|S| \leq k \text { ? }\end{array}$ \\
\hline$\gamma(G)$ & $\begin{array}{l}\text { LOWER DOMINATION } \\
\text { MINIMUM DOMINATING SET }\end{array}$ & $\begin{array}{l}\text { Does } G \text { have a } \\
\text { dominating set } D \\
\text { such that }|D| \leq k \text { ? }\end{array}$ \\
\hline$i(G)$ & $\begin{array}{l}\text { LOWER INDEPENDENCE } \\
\text { MINIMUM INDEPENDENT DOMINATING SET } \\
\text { MINIMUM MAXIMAL INDEPENDENT SET }\end{array}$ & $\begin{array}{l}\text { Does } G \text { have a maximal } \\
\text { independent set } I \\
\text { such that }|I| \leq k \text { ? }\end{array}$ \\
\hline$\alpha(G)$ & $\begin{array}{l}\text { UPPER INDEPENDENCE } \\
\text { MAXIMUM INDEPENDENT SET }\end{array}$ & $\begin{array}{l}\text { Does } G \text { have an } \\
\text { independent set } I \\
\text { such that }|I| \geq k \text { ? }\end{array}$ \\
\hline$\Gamma(G)$ & $\begin{array}{l}\text { UPPER DOMINATION } \\
\text { MAXIMUM MINIMAL DOMINATING SET }\end{array}$ & $\begin{array}{l}\text { Does } G \text { have a minimal } \\
\text { dominating set } D \\
\text { such that }|D| \geq k \text { ? }\end{array}$ \\
\hline $\operatorname{IR}(G)$ & $\begin{array}{l}\text { UPPER IRREDUNDANCE } \\
\text { MAXIMUM IRREDUNDANT SET }\end{array}$ & $\begin{array}{l}\text { Does } G \text { have an } \\
\text { irredundant set } S \\
\text { such that }|S| \geq k \text { ? }\end{array}$ \\
\hline
\end{tabular}

such that removing all the vertices of the outer face results in a $k$-1-outerplanar graph. For every planar graph $G$ there is some $k$, such that $G$ is a $k$-outerplanar. There is a polynomial time algorithm that can take as input a planar graph $G$, and produce a $k$-outerplanar embedding of $G$ for which $k$ is minimum [18].

For any $0<\varepsilon<1-\frac{1}{n}$, a graph $G=(V, E)$ with $|V|=n$ is called everywhere- $\varepsilon$-dense if every vertex in $G$ has at least $\varepsilon|V|$ neighbours; similarly, for every $0<\varepsilon<\frac{1}{2}-\frac{1}{2 n}$, a graph $G=(V, E)$ with $|V|=n$ is called average- $\varepsilon$-dense if $|E| \geq \varepsilon n^{2}$.

For any set $S \subseteq V$ and $v \in S$ we define the private neighbourhood of $v$ with respect to $S$ as $p n(v, S)=N[v]-N[S-\{v\}]$. Any $w \in \operatorname{pn}(v, S)$ is called a private neighbour of $v$ with respect to $S$. We sometimes omit "with respect to $S$ " if $S$ is clear from the context.

Independence and domination in graphs are very well known and studied concepts in graph algorithms, while irredundance is much less investigated. With the notion of private neighbourhoods, irredundance means that each vertex has a private neighbour; formally, $S \subseteq V$ is an irredundant set if and only if for each vertex $v \in S,|p n(v, S)|>0$.

In Table 2, we define the six problems of finding the six parameters in the domination chain for a given graph $G$.

The domination chain is largely due to the following combinatorial properties:

1. Every maximal independent set is a minimal dominating set [13].

2. A dominating set $D$ is minimal if and only if $|p n(v, D)|>0$ for every $v \in D$ [33].

Observe that $v$ can be a private neighbour of itself, i.e., a dominating set is minimal if and only if it is also an irredundant set. Actually, every minimal dominating set is also a maximal irredundant set [21].

Given a graph $G=(V, E)$, we use $\zeta(G)$ to denote any of the domination chain parameters in Table 1, where $\zeta(G) \in$ $\{\operatorname{ir}(G), \gamma(G), i(G), \alpha(G), \Gamma(G), \operatorname{IR}(G)\}$. We define co $-\zeta(G)=n(G)-\zeta(G)$. Then, we can state the following complementary domination chain:

$$
\mathrm{co}-\operatorname{IR}(G) \leq \mathrm{co}-\Gamma(G) \leq \mathrm{co}-\alpha(G) \leq \mathrm{co}-i(G) \leq \mathrm{co}-\gamma(G) \leq \mathrm{co}-\operatorname{ir}(G) .
$$

Sometimes, the complement problems have received their own names, such as Nonblocker [38], Maximum ENCLAVELESS Set [70], or Maximum Spanning StaR Forest [9,66], which all refer to the complement problem of Minimum Domination. The probably most studied among these problems is Minimum VerTEX COVER which refers to the complement problem of MAXImum InDEPENDENT SET. We will also use $\tau(G)$ instead of co $-\alpha(G)$ to refer to this graph parameter.

In Table 3, we define the six problems of finding the six co-parameters in the complementary domination chain for a given graph G.

For the basic definitions on classical complexity, approximation and parameterised algorithms we refer to standard texts like $[10,41]$. As most of our results are on approximation complexity, we shortly revise some basic notions.

Given an optimisation problem in NPO and an instance $I$ of this problem, we use $|I|$ to denote the size of $I$, opt $(I)$ to denote the optimum value of $I$, and $\operatorname{val}(I, S)$ to denote the value of a feasible solution $S$ of instance $I$. The performance ratio of $S$ (or approximation factor) is $r(I, S)=\max \left\{\frac{\operatorname{val}(I, S)}{\operatorname{opt}(I)}, \frac{\operatorname{opt}(I)}{\operatorname{val}(I, S)}\right\}$. The error of $S, \varepsilon(I, S)$, is defined by $\varepsilon(I, S)=r(I, S)-1$. 
Table 3

The six problems in the complementary domination chain. The input is the same for all the problems: a graph $G(V, E)$ and a non-negative integer $k$. For each problem, the alternative name used in this paper is given in bold.

\begin{tabular}{|c|c|c|}
\hline Parameter & $\begin{array}{l}\text { Problem } \\
\text { (Alternative names) }\end{array}$ & Question \\
\hline co $-\operatorname{ir}(G)$ & $\begin{array}{l}\text { Co-LOWER IRREDUNDANCE } \\
\text { MAXIMUM MINIMAL Co-IRREDUNDANT SET }\end{array}$ & $\begin{array}{l}\text { Does } G \text { have a maximal } \\
\text { irredundant set } S \\
\text { such that }|S| \leq n-k \text { ? }\end{array}$ \\
\hline $\mathrm{co}-\gamma(G)$ & $\begin{array}{l}\text { Co-LOWER DOMINATION } \\
\text { MAXIMUM Co-DOMINATING SET } \\
\text { NONBLOCKER } \\
\text { MAXIMUM ENCLAVELESS SET } \\
\text { MAXIMUM SPANNING STAR FOREST }\end{array}$ & $\begin{array}{l}\text { Does } G \text { have a } \\
\text { dominating set } D \\
\text { such that }|D| \leq n-k \text { ? }\end{array}$ \\
\hline co $-i(G)$ & $\begin{array}{l}\text { Co-LoWER INDEPENDENCE } \\
\text { MAXimum Minimal Co-INDEPENDENT Set } \\
\text { MaXimum Minimal VerTeX Cover }\end{array}$ & $\begin{array}{l}\text { Does } G \text { have a maximal } \\
\text { independent set } I \\
\text { such that }|I| \leq n-k \text { ? }\end{array}$ \\
\hline $\begin{array}{l}\text { со }-\alpha(G) \\
=\tau(G)\end{array}$ & $\begin{array}{l}\text { CO-UPPER INDEPENDENCE } \\
\text { MINIMUM CO-INDEPENDENT SET } \\
\text { MINIMUM VERTEX COVER }\end{array}$ & $\begin{array}{l}\text { Does } G \text { have an } \\
\text { independent set } I \\
\text { such that }|I| \geq n-k \text { ? }\end{array}$ \\
\hline $\mathrm{co}-\Gamma(G)$ & $\begin{array}{l}\text { Co-UPPER DOMINATION } \\
\text { Minimum MAXIMAL Co-DOMINATING SET }\end{array}$ & $\begin{array}{l}\text { Does } G \text { have a minimal } \\
\text { dominating set } D \\
\text { such that }|D| \geq n-k \text { ? }\end{array}$ \\
\hline co - IR $(G)$ & $\begin{array}{l}\text { CO-UPPER IRREDUNDANCE } \\
\text { MINIMUM CO-IRREDUNDANT SET }\end{array}$ & $\begin{array}{l}\text { Does } G \text { have an } \\
\text { irredundant set } S \\
\text { such that }|S| \geq n-k \text { ? }\end{array}$ \\
\hline
\end{tabular}

For a function $f$, an algorithm is an $f(|I|)$-approximation, if for every instance $I$ of the problem, it returns a solution $S$ such that $r(I, S) \leq f(|I|)$.

For providing hardness proofs in the area of approximation algorithms, L-reductions and E-reductions have become the de facto standard. We use an approximation-preserving reduction, called L-reduction, introduced by Papadimitriou and Yannakakis in [67], in order to show that a problem does not admit a PTAS (polynomial-time approximation scheme). Let $A$ and $B$ be two optimisation problems. Then $A$ is said to be $L$-reducible to $B$ if there exist polynomial time computable functions $f, g$ and two constants $a, b>0$ such that

1. $f$ maps an instance $I$ of $A$ to an instance $f(I)$ of $B$ such that $o p t_{B}(f(I)) \leq a \cdot o p t_{A}(I)$ for all instances $I$ of $A$.

2. $g$ maps for any instance $I$ of $A$ a solution $S^{\prime}$ for instance $f(I)$ to a solution $S$ for $I$ such that $\left|v a l(I, S)-o p t_{A}(I)\right| \leq$ $b \cdot\left|\operatorname{val}\left(f(I), S^{\prime}\right)-o p t_{B}(f(I))\right|$.

For us, the important property of this reduction is that if $A$ is $L$-reducible to $B$ and $A$ has no PTAS then $B$ has no PTAS.

The notion of an E-reduction (error-preserving reduction) was introduced by Khanna et al. [57]. A problem $A$ is called $E$-reducible to a problem $B$, if there exist polynomial time computable functions $f, g$ and a constant $b>0$ such that

- $f$ maps an instance $I$ of $A$ to an instance $f(I)$ of $B$ such that opt $(I)$ and $\operatorname{opt}(f(I))$ are related by a polynomial factor, i.e. there exists a polynomial $p$ such that $\operatorname{opt}(f(I)) \leq p(|I|) \cdot \operatorname{opt}(I)$.

- $g$ maps for any instance $I$ of $A$ any solution $S^{\prime}$ of $f(I)$ to a solution $S$ of $I$ such that $\varepsilon(I, S) \leq b \cdot \varepsilon\left(f(I), S^{\prime}\right)$.

An important property of an E-reduction is that it can be applied uniformly to all levels of approximability; that is, if $A$ is $E$-reducible to $B$ and $B$ belongs to $\mathcal{C}$ then $A$ belongs to $\mathcal{C}$ as well, where $\mathcal{C}$ is a class of optimisation problems with any kind of approximation guarantee (see also [57]).

\subsection{Previous work/main contributions and organisation of the paper}

Our main contributions and the paper organisation are as follows.

- In this subsection we give a summary of the complexity results for the six problems in the domination chain, both for the previous results and the ones proved in this paper. We include results on exact, parameterised and approximation algorithms, as well as (in)approximability results and special classes of graphs. For readability, we summarise all the results in tables and for each result we provide either a reference to another paper or a theorem (corollary, observation) in this paper.

- In Section 2, we give a brief overview of the known relationships between the parameters of the domination chain. We then analyse the structure of maximum irredundant sets and provide new bounds on the upper irredundance number $\operatorname{IR}(G)$ in terms of $\alpha(G)$. 
Table 4

Table of known results for general graphs on the six problems in the domination chain.

\begin{tabular}{|c|c|c|c|c|c|c|}
\hline \multirow[b]{2}{*}{ ir } & \multicolumn{2}{|l|}{$\begin{array}{l}\text { Exact algorithm } \\
\text { w.r.t } n=|V|\end{array}$} & \multicolumn{2}{|c|}{$\begin{array}{l}\text { Parameterised Compl. } \\
\text { standard parameterisation }\end{array}$} & \multicolumn{2}{|c|}{$\begin{array}{l}\text { Tight Inapprox. ratio } \\
\text { under assumption } \mathrm{P} \neq \mathrm{NP}\end{array}$} \\
\hline & $\mathcal{O}^{*}\left(1.99914^{n}\right)$ & [19] & W[2]-complete & [19] & $c \log (n)$ & Proposition 13 \\
\hline$\gamma$ & $\mathcal{O}^{*}\left(1.4864^{n}\right)$ & [55] & W[2]-complete & [40] & $c \log (n)$ & {$[39,44,65]$} \\
\hline$i$ & $\mathcal{O}^{*}\left(1.3351^{n}\right)$ & [23] & W[2]-complete & [40] & $n^{1-\varepsilon}$ & {$[52]$} \\
\hline$\alpha$ & $\mathcal{O}^{*}\left(1.1996^{n}\right)$ & [71] & W[1]-complete & [40] & $n^{1-\varepsilon}$ & [72] \\
\hline$\Gamma$ & $\mathcal{O}^{*}\left(1.7159^{n}\right)$ & [12] & W[1]-hard, in W[2] & [12] & $n^{1-\varepsilon}$ & [12] \\
\hline IR & $\mathcal{O}^{*}\left(1.9369^{n}\right)$ & [19] & W[1]-complete & [42] & $n^{1-\varepsilon}$ & Theorem 14 \\
\hline
\end{tabular}

Table 5

Table of (currently best known) results for the six problems in the domination chain on graphs with degree restrictions, where $\Delta$ denotes the maximum degree and dense denotes everywhere- $\varepsilon$-dense.

\begin{tabular}{|c|c|c|c|c|c|}
\hline & Vertex-kernel & Apx-ratio & $\begin{array}{l}\text { APX-C } \\
\Delta=3\end{array}$ & $\begin{array}{l}\text { NP-C } \\
\text { planar cubic }\end{array}$ & $\begin{array}{l}\text { Non-apx } \\
\text { dense }\end{array}$ \\
\hline ir & $\begin{array}{l}\frac{3}{2} \Delta k \\
\text { (Observation 26) }\end{array}$ & $\begin{array}{l}\frac{3}{2} \Delta \\
{[35]}\end{array}$ & (Corollary 29) & (Theorem 20) & $?$ \\
\hline$\gamma$ & $\begin{array}{l}(\Delta+1) k \\
\text { (Observation 25) }\end{array}$ & $\begin{array}{l}\log (\Delta)+1 \\
{[30]}\end{array}$ & [3] & [48] & $\begin{array}{l}\text { APX-H on } \\
\text { avg dense } \\
{[50]}\end{array}$ \\
\hline$i$ & $\begin{array}{l}(\Delta+1) k \\
\text { (Observation 25) }\end{array}$ & $\begin{array}{l}\Delta+1 \\
\text { (Observation 25) }\end{array}$ & (Corollary 31) & [62] & $\begin{array}{l}n^{1-\varepsilon} \\
\text { (Theorem 36) }\end{array}$ \\
\hline$\alpha$ & $\begin{array}{l}\Delta k \\
\text { (Observation 24) }\end{array}$ & $\begin{array}{l}\frac{\Delta+3}{5} \\
{[14]}\end{array}$ & [3] & [47] & $\begin{array}{l}n^{1-\varepsilon} \\
\text { (Proposition 35) }\end{array}$ \\
\hline$\Gamma$ & $\begin{array}{l}\Delta k \\
\text { (Observation 24) }\end{array}$ & $\begin{array}{l}\frac{6 \Delta^{2}+2 \Delta-3}{10 \Delta} \\
{[12]}\end{array}$ & {$[12]$} & [12] & $\begin{array}{l}n^{1-\varepsilon} \\
\text { (Corollary 34) }\end{array}$ \\
\hline IR & $\begin{array}{l}\Delta k \\
\text { (Observation 24) }\end{array}$ & $\begin{array}{l}\frac{6 \Delta^{2}+2 \Delta-3}{10 \Delta} \\
\text { (Observation 23) }\end{array}$ & (Corollary 30 ) & (Theorem 21) & $\begin{array}{l}n^{1-\varepsilon} \\
\text { (Theorem 33) }\end{array}$ \\
\hline
\end{tabular}

- In Section 3, we give several (in)approximability results on general graphs, for lower and upper irredundance and their complements.

- In Section 4, we prove NP-hardness of the lower and upper irredundance problem on planar cubic graphs, which also implies the hardness of the complement problems on planar cubic graphs.

- In Section 5 we consider graphs with bounded degree. We show APX-hardness on cubic graphs for minimum domination, minimum independent domination, and lower and upper irredundance, as well as the their complements. We also provide some simple algorithmic results to complement the hardness results.

- In Section 6, we present some approximability results on everywhere dense graphs, for upper irredundance, upper domination, minimum maximal independent set and their complements.

- In Section 7, we study some less known problems related to the domination chain, and we show that both MiNIMAL Vertex Cover Extension, and the Minimal Co-IRREdundant Extension are NP-hard, the former even when restricted to planar cubic graphs.

- Finally in Section 8, we give some concluding remarks and open problems.

In order to get our results we mainly establish polynomial reductions between decision versions and L and E-reductions between optimisation problems.

In Tables 4-6, we summarise the known algorithmic and complexity aspects of the domination chain parameters, as well as some of the new results obtained in this paper. Clearly, the classical complexity results for the problems of the domination chain in Tables 4 and 5 also apply to their complements and are hence not repeated in Table 6. However, observe that the status of parameterised complexity and approximation of these problems and their complementary versions indeed differ. In order to distinguish the problem parameters of the two tables, we use $k$ in Tables 4,5 and $\ell$ in Table 6. 
Table 6

Table of (currently best known) results for the six problems in the complementary domination chain, where $\ell$ denotes the standard parameter (bound on the optimal solution) and dense denotes everywhere- $\varepsilon$-dense.

\begin{tabular}{|c|c|c|c|c|c|}
\hline & Apx-ratio & Non-Apx & Vertex-kernel & $\begin{array}{l}\text { FPT } \\
\mathcal{O}^{*}-\mathrm{Alg}\end{array}$ & $\begin{array}{l}\text { APX-H } \\
\text { dense }\end{array}$ \\
\hline co $-\operatorname{ir}(G)$ & 2 (Observation 15) & $?$ & $2 \ell-1[19]$ & $3.841^{\ell}[19]$ & $?$ \\
\hline co $-\gamma(G)$ & $\frac{240}{193}[8]$ & $\frac{260}{259}[66]$ & $\frac{5}{3} \ell+3[38]$ & $2.0226^{\ell}[38]$ & $?$ \\
\hline $\mathrm{co}-i(G)$ & $\sqrt{n}[22]$ & $n^{\frac{1}{2}-\varepsilon}[22]$ & $\ell^{2}[45]($ Sec.4.3) & $1.5874^{\ell}[22]$ & (Theorem 36) \\
\hline co $-\alpha(G)$ & 2 (folklore) & 2 (UGC) [58] & $2 \ell[27,41]$ & $1.2738^{\ell}[29]$ & {$[31]$} \\
\hline co $-\Gamma(G)$ & $4[12]$ & $?$ & $\ell^{2}+\ell[12]$ & $4.3077^{\ell}[12]$ & (Corollary 34) \\
\hline co $-\operatorname{IR}(G)$ & 4 (Theorem 16) & $?$ & $3 \ell[19]$ & $2.8752^{\ell}[19]$ & (Theorem 33) \\
\hline
\end{tabular}

\section{Notes on the structure of the maximal irredundant set, and the relationship between the domination chain parameters}

There are several known relationships between the parameters of the domination chain. The next theorem relates the lower irredundance number to the (lower) domination number.

Theorem 3 ([21]). For any graph G:

$$
\gamma(G) \leq 2 \operatorname{ir}(G)-1 \text {. }
$$

The previous bound was further strengthened in [5] to $\gamma(G) \leq 2 \operatorname{ir}(G)-(k+1)$, where $k$ is the maximum number of vertices $v$ in a minimum irredundant set $S$ with $v \in p n(v, S)$ (there referred to as isolates in $\operatorname{ir}(G)$ ).

The following theorem relates the (lower) domination and the lower independence numbers of graphs with no isolated vertices.

Theorem 4 ([21]). For any graph $G$ with minimum degree $\delta(G) \geq 1$ :

$$
i(G) \leq n-\gamma(G)+1-\left\lceil\frac{n-\gamma(G)}{\gamma(G)}\right\rceil .
$$

The following theorem provides a stronger result for claw-free graphs.

Theorem 5 ([4]). For any graph $G$ that does not have an induced subgraph isomorphic to $K_{1,3}$ :

$$
\gamma(G)=i(G) .
$$

Finally, the following two theorems relate the upper domination number to the (upper) independence number of a graph.

Lemma 6 ([12]). For any connected graph $G$ with $n>0$ vertices we have:

$$
\alpha(G) \leq \Gamma(G) \leq \max \left\{\alpha(G), \frac{n}{2}+\frac{\alpha(G)}{2}-1\right\}
$$

Lemma 7 ([12]). For any connected graph $G$ with $n>0$ vertices, minimum degree $\delta$ and maximum degree $\Delta$, we have:

$$
\alpha(G) \leq \Gamma(G) \leq \max \left\{\alpha(G), \frac{n}{2}+\frac{\alpha(G)(\Delta-\delta)}{2 \Delta}-\frac{\Delta-\delta}{\Delta}\right\} .
$$

In this paper, we show that the bounds on $\Gamma$ given by Lemmas 6 and 7 also hold for the upper irredundance number IR. In order to do that, we first need to analyse the structure of maximal irredundant sets in a graph.

Any maximal irredundant set $S$ for a graph $G=(V, E)$ can be associated with a partition of the set of vertices $V$ into five sets $F, I, P, O, R$ given by: $I:=\{v \in S: v \in p n(v, S)\}, F:=S-I, P \in\{B \subseteq N(F) \backslash S:|p n(v, S) \cap B|=1$ for all $v \in F\}$ with $|F|=|P|, O=N[S]-(S \cup P), R=V-N[S]$. For an illustration of this partition, see Fig. 1. This representation is not necessarily unique since there might be different choices for $P$, but for every partition of this kind, the following properties hold:

1. Every vertex $v \in F$ has at least one neighbour in $F$, called a friend.

2. The set $I$ is an independent set in $G$. 


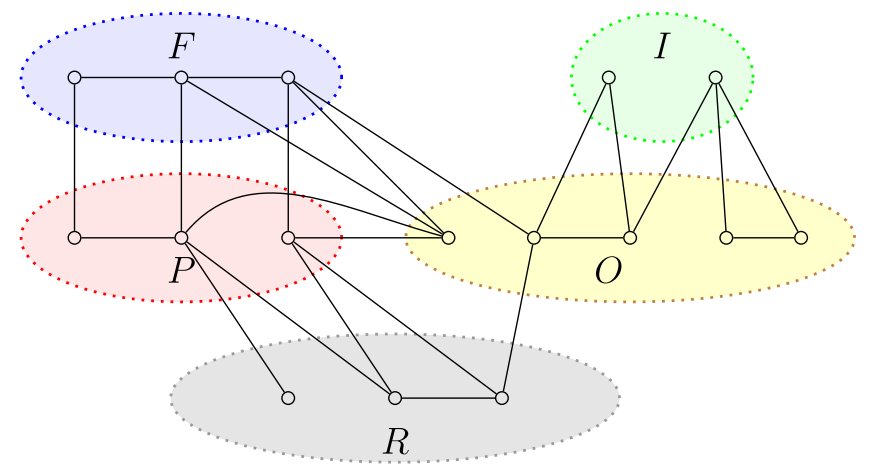

Fig. 1. Illustration of a partition of a maximal irredundant set into the sets $F, I, P, O, R$.

3. The subgraph induced by the vertices $F \cup P$ has an edge cut set separating $F$ and $P$ that is, at the same time, a perfect matching; hence, $P$ can serve as the set of private neighbours for $F$.

4. The neighbourhood of a vertex in $I$ is always a subset of $O$, which are otherwise the outsiders.

5. Each vertex in $R$ has at least one neighbour in $P$.

For property 5, observe that if there is a vertex $v \in R$ which does not have a neighbour in $P$, then $S \cup\{v\}$ is also an irredundant set since no private neighbourhood is empty:

- $v \in p n(v, S \cup\{v\})$ as $v$ was not in $O$, hence not adjacent to any vertex in $S$,

- for all $w \in I, w$ remains in its own private neighbourhood, as $v \in R$ is not adjacent to any $w \in I$,

- for all $w \in F$, their respective private neighbour in $P$ remains private, as $v$ is not adjacent to it.

Consequently, any maximal irredundant set has to satisfy property 5 .

Lemma 8. For any connected graph $G$ with $n>0$ vertices and a maximum irredundant set $S$ with an associated partition $(F, I, P, O, R)$ as defined above, if $|S|=\operatorname{IR}(G)>\alpha(G)$ then $|I| \leq \alpha(G)-2$.

Proof. Let $G$ be a connected graph with $n>0$ vertices and let $S$ be a maximum irredundant set with an associated partition $(F, I, P, O, R)$. We first show that if $I R>\alpha(G)$ then $|F| \geq 2$ (in fact, one can show that then $|F| \geq 3$ but that is not necessary for our proof). Indeed, if $|F|=0$, then $S$ is also an independent set, and thus $\operatorname{IR}(G)=\alpha(G)$, and according to our definition of partition $(F, I, P, O, R)$, we have $|F| \neq 1$ (see Property 1 of this partition).

Now, if $|F| \geq 2$ then the subgraph of $G$ induced by $F \cup P$ contains an independent set of size 2 consisting of a vertex in $F$, say $v$, and a vertex in $P$, say $u$, such that $v$ and $u$ are not adjacent. Since in the original graph $G$, there are no edges between the vertices in $I$ and the vertices in $F \cup P$ (Property 4), $I \cup\{u, v\}$ forms an independent set of size $|I|+2$. This sets a lower bound on the independence number and we have $\alpha(G) \geq|I|+2$.

From the above, it follows that if $\operatorname{IR}(G)>\alpha(G)$ then $|I| \leq \alpha(G)-2$.

Lemma 9. For any connected graph $G$ with $n>0$ vertices we have:

$$
\alpha(G) \leq \operatorname{IR}(G) \leq \max \left\{\alpha(G), \frac{n}{2}+\frac{\alpha(G)}{2}-1\right\} .
$$

Proof. We consider a graph $G$ with $n>0$ vertices and let $S$ be a maximum irredundant set with associated partition $(F, I, P, O, R)$. We examine separately the following two cases:

1. $\operatorname{IR}(G)=\alpha(G)$. Then we trivially have $\operatorname{IR}(G) \leq \alpha(G)$.

2. $\operatorname{IR}(G)>\alpha(G)$.

From the fact that $|F|=|P|$ (from Property 3) we have $|F|=\frac{n-|I|-|O|-|R|}{2} \leq\left\lfloor\frac{n-|I|}{2}\right\rfloor$ and thus

$$
\operatorname{IR}(G)=|F|+|I| \leq\left\lfloor\frac{n+|I|}{2}\right\rfloor .
$$

From the above and Lemma 8 we have

$$
\operatorname{IR}(G) \leq\left\lfloor\frac{n+|I|}{2}\right\rfloor \leq\left\lfloor\frac{n+\alpha(G)-2}{2}\right\rfloor \leq \frac{n}{2}+\frac{\alpha(G)}{2}-1 .
$$

This concludes the proof of the claim. 
Lemma 10. For any connected graph $G$ with $n>0$ vertices, minimum degree $\delta$ and maximum degree $\Delta$, we have:

$$
\alpha(G) \leq \operatorname{IR}(G) \leq \max \left\{\alpha(G), \frac{n}{2}+\frac{\alpha(G)(\Delta-\delta)}{2 \Delta}-\frac{\Delta-\delta}{\Delta}\right\} .
$$

Proof. Let $G$ be a connected graph with $n>0$ vertices, maximum degree $\Delta$ and let $S$ be a maximum irredundant set with associated partition $(F, I, P, O, R)$. Our argument is similar to the one in Lemma 9 , as the case $\operatorname{IR}(G)=\alpha(G)$ is again trivial, assume $\operatorname{IR}(G)>\alpha(G)$. Again, we obtain:

$$
\operatorname{IR}(G)=|F|+|I|=\frac{n+|I|-|O|-|R|}{2} .
$$

We next derive an improved lower bound on $|O|$. Let $e$ be the number of edges adjacent with vertices from $I$. As $G$ is of minimum degree $\delta$, we have $e \geq \delta|I|$. As the vertices in $I$ are only adjacent with the vertices in $O$, there are at least $e$ edges that have exactly one end vertex in $O$. Since $G$ has maximum degree $\Delta$, we have that $|O| \geq\left\lceil\frac{e}{\Delta}\right\rceil \geq\left\lceil\frac{\delta[I]}{\Delta}\right\rceil$.

From the above and Lemma 8 we have

$$
\begin{aligned}
\operatorname{IR}(G) & \leq\left\lfloor\frac{n+|I|-\left\lceil\frac{\delta|I|}{\Delta}\right\rceil-|R|}{2}\right\rfloor \leq \frac{n+|I|-\frac{\delta|I|}{\Delta}}{2}=\frac{n+\frac{(\Delta-\delta)|I|}{\Delta}}{2} \\
& \leq \frac{n+\frac{(\Delta-\delta)}{\Delta}(\alpha(G)-2)}{2}=\frac{n}{2}+\frac{\Delta-\delta}{2 \Delta} \alpha(G)-\frac{\Delta-\delta}{\Delta} .
\end{aligned}
$$

This lemma generalises [54, Proposition 12], which states the property for $\Delta$-regular graphs, where, in particular, $\delta=\Delta$. Eq. (7) immediately yields:

Lemma 11. Let $G$ be a connected graph. Then,

$$
\frac{\tau(G)}{2}+1 \leq \operatorname{co}-\operatorname{IR}(G) \leq \tau(G)
$$

We also like to point the reader to [73], where another interesting combinatorial bound was shown, namely

$$
\operatorname{IR}(G)-\alpha(G) \leq\left\lceil\frac{\Delta-2}{2 \Delta} n\right\rceil .
$$

\section{Approximability on general graphs}

In this section, we discuss approximability on general graphs. For some problems from the domination chain, the assumption $\mathrm{P} \neq \mathrm{NP}$ yields strong inapproximability results; more precisely, it is known that under this assumption there is no $n^{1-\varepsilon}$-approximation for Minimum InDEPENDENT Domination [52], MaXimum INDEPENDENT Set [72] and UPPER DOMINATION [12]. We will show in this section that this inapproximability also holds for UPPER IRREDUNDANCE but first we like to comment on the status of the inapproximability of Minimum Domination.

Remark 12. There seems to be no explicit reference for the inapproximability of Minimum Domination, at least under the assumption $\mathrm{P} \neq \mathrm{NP}$. The standard reference is to Feige [44], but even this is a bit indirect, as he only considers SET COVER explicitly, but as the sizes of the universe and of the set system are polynomially related, the standard reduction from SET Cover to Minimum Domination gives the desired inapproximability result, yet under a non-standard complexity assumption. Dinur and Steuer developed techniques further and showed [39] that SET COVER is hard to approximate within some logarithmic factor under the assumption that $\mathrm{P} \neq \mathrm{NP}$. From the proof given by Moshkovitz [65], it becomes clear that the sizes of the universe and of the set system are polynomially related, so that the claimed inapproximability result for Minimum Domination can be deduced. More specifically, the proof is via LABEL Cover, where instances are given as bipartite graphs $(A, B ; E)$. Now, the number of elements in the derived SET Cover instance is a small polynomial in $|B|$, and the number of sets is a small polynomial in $|A|$, and the degree of the vertices in $B$ is bounded by some constant $D$. In particular, the number of sets in the constructed SET COVER instance is bounded by a polynomial in the number of elements of that instance.

The standard reduction from Set Cover to Minimum Domination creates a split-graph as an instance for Minimum Domination. Since $\operatorname{ir}(G)=\gamma(G)$ for all split-graphs $G$ by [60], this standard reduction also yields:

Proposition 13. For any $\varepsilon>0$, there is no $(1-\varepsilon) \log (n)$-approximation for LowER IRREDUNDANCE, where $n$ is the number of vertices of the input graph, unless $\mathrm{P}=\mathrm{NP}$, even when the inputs are restricted to split graphs.

The inapproximability for Minimum Domination is tight as it is well known that a greedily build minimum dominating set yields an approximation with a ratio in $\mathcal{O}(\log (n))$. Eq. (2) together with the relation $\operatorname{ir}(G) \leq \gamma(G)$ and the fact that each 
minimal dominating set is a minimal irredundant set transfers this approximability of MiNIMUM DoMINATION to LOWER IRREDUNDANCE.

For UPPER IRREDUNDANCE we consider induced matchings to investigate approximability. An induced matching of a graph $G=(V, E)$ is a subset $M \subseteq V$ such that all vertices in the induced subgraph $G[M]$ have degree one. We consider the following optimisation problem in this context:

\begin{tabular}{||c|c|c||}
\hline \hline Parameter & Problem & Question \\
\hline $\operatorname{im}(G)$ & MAXIMUM InducED MATCHING & $\begin{array}{c}\text { Does } G \text { have an induced } \\
\text { matching } M \text { with }|M| \geq k ?\end{array}$ \\
\hline \hline
\end{tabular}

In [25], Chalermsook et al. state that there is no $|V|^{1-\varepsilon}$-approximation for Maximum InduCED Matching even when restricted to bipartite graphs, unless NP $\subseteq$ ZPP. Their reduction yields this hardness by a gap-preserving reduction from MAXIMUM INDEPENDENT SET. In combination with the approximation-hardness from [72] for MAXIMUM INDEPENDENT SET this reduction also yields the stated inapproximability under the stronger assumption $\mathrm{P} \neq \mathrm{NP}$.

Theorem 14. For any $\varepsilon>0$, UPPER IRREDUNDANCE is not $n^{1-\varepsilon}$-approximable, where $n$ is the number of vertices of the input graph, unless $\mathrm{P}=\mathrm{NP}$, even when the inputs are restricted to co-bipartite graphs.

Proof. Given a bipartite graph $G=\left(V_{1}, V_{2}, E\right)$ as input for Maximum Induced Matching restricted to bipartite graphs, we construct an instance $G^{\prime}$ of UPPER IRREDUNDANCE, simply by turning both $V_{1}$ and $V_{2}$ into cliques. Any two vertices $u \in V_{1}$ and $v \in V_{2}$ are already dominating for $G^{\prime}$, which implies that any irredundant set $S$ for $G^{\prime}$ which has cardinality more than two is completely contained in either $V_{1}$ or $V_{2}$. In any associated partition $(F, I, P, O, R), I$ is empty and $S$ is consequently equal to $F$. Since $F \cup P$ has a matching cut in $G^{\prime}$ by definition of the partition $(F, I, P, O, R)$ it is clear that $F \cup P$ is an induced matching in $G$ which yields $\operatorname{im}(G) \geq 2 \operatorname{IR}\left(G^{\prime}\right)$.

Conversely, consider any induced matching $M$ in $G$. For the set $S=M \cap V_{1}$, we know that each vertex in $S$ has exactly one neighbour in $M$ and that this neighbour is not adjacent to any other vertex in $S$. In the graph $G^{\prime}$, this implies that each vertex in $S$ has at least its matched neighbour from $M$ as private neighbour, which means that $S$ is irredundant in $G^{\prime}$ and hence $\operatorname{im}(G) \leq 2 \operatorname{IR}\left(G^{\prime}\right)$.

Overall, we have and E-reduction since $\operatorname{im}(G)=2 \operatorname{IR}\left(G^{\prime}\right)$ and $\left|V_{G}\right|=\left|V_{G^{\prime}}\right|$, which transfers the approximation-hardness from MAXIMum InducED MATCHING restricted to bipartite graphs to UPPER IRREDUNDANCE (even restricted to co-bipartite graphs).

As an interesting aside, let us mention that the relation between UPPER IRREDUNDANCE and MAXIMUM INDUCED MATCHING is also useful in a reverse direction. Namely, in [19, Sec. 6], the exact algorithm developed for UPPER IRREDUNDANCE was obtained by first translating the graph into an instance of MAXIMUM INDUCED MATCHING on bipartite graphs (called BIPARTITE INDUCED MATCHING in that paper) and then solving the parameterised version of that problem in time $O^{*}\left(1.7^{k}\right)$.

For the little studied complement of LoWER IRREDUNDANCE we observe:

Observation 15. For any graph $G$ without isolated vertices one can compute a minimal dominating set of cardinality at most $\frac{n}{2}$ in polynomial time by computing Minimum Domination for an arbitrary spanning forest of $G$. The complement of this dominating set is consequently a 2-approximation for Co-LOWER IRREDUNDANCE.

Using Lemma 11, one can use known exact or approximation algorithms for Minimum VerTex Cover to deduce:

Theorem 16. Co-UPPER IRREDUNDANCE is 4-approximable in polynomial time and 2-approximable in $0^{*}\left(1.2738^{\tau(G)}\right)$ or $0^{*}\left(1.1996^{n}\right)$ time.

Proof. Given a graph $G$ on $n$ vertices, we first find a vertex cover $C$ in $G$ using any 2-approximation algorithm, and define $S^{\prime}=V \backslash C$. Set $S^{\prime}$ is an independent set and let $S$ be a maximal independent set containing $S^{\prime}$. The set $V \backslash S$ is a vertex cover of size $|V \backslash S| \leq|C| \leq 2 \tau(G) \leq 4(n-\operatorname{IR}(G))$, see Lemma 11. Moreover, $V \backslash S$ is the complement of a maximal independent set which also makes it the complement of a maximal irredundant set, so overall a feasible solution for CO-UPPER IRREDUNDANCE with $|V \backslash S| \leq 4(n-\operatorname{IR}(G))$. The claimed running time for the factor- 2 approximation stems from the best parameterised and exact algorithms for Minimum VerTex Cover by [29] and [71].

We could also use some results from parameterised approximation. For instance, by [24], we can conclude:

Corollary 17. CO-UPPER IRREDUNDANCE is 3-approximable in time $0^{*}\left(1.0883^{\tau(G)}\right)$. 


\section{Planar graphs}

While approximability and fixed parameter tractability for all problems of the domination chain are rather unsuccessful, the situation improves for the restriction to planar graphs.

It is known that any planar graph $G$ has treewidth in $\mathcal{O}(\sqrt{\gamma(G)})$ [2] which by Eq. (2) means that the treewidth can also be bounded by $\operatorname{ir}(G)$. Since the properties irredundance, independence and domination can all be expressed in monadic second order logic, all problems of the domination chain are in FPT for the restriction to planar graphs by Courcelle's theorem [36].

In combination with the concept of outerplanarity, these results for bounded treewidth can be used to develop PTAS as initially shown for a series of problems including MAXIMum InDEPendent Set and Minimum Vertex Cover in [11]. There it was also sketched how to solve Minimum Domination same way as Minimum Vertex Cover; this idea however turned out to need more adjustment and a detailed approach with the flaws in the initial sketch is shown in [63]. In [12] it is shown that this concept can also be adapted for UPPER DOMINATION. The problematic part for this approximation scheme is to show that dominating the separators can be done without eliminating too many private neighbourhoods which requires a careful analysis.

It is not hard to see that the approach from [12] can also be adapted for UPPER IRREDUNDANCE without these problems.

Proposition 18. UPPER IRREDUNDANCE admits a PTAS on planar graphs.

Proof. The approximation scheme is built as usual within Baker's approach [11]. We only explain the main ingredients in the following. (a) Observe that given two irredundant sets $S_{1}$ and $S_{2}$ for two vertex-disjoint subgraphs $G_{1}$ and $G_{2}$ of $G$, respectively (where 'disjoint' here means that the vertices from $G_{1}$ are in $G$ not adjacent to the vertices from $G_{2}$ ), the set $S_{1} \cup S_{2}$ is irredundant in $G$ and can greedily be extended to a maximal irredundant set for $G$. (b) Conversely, given any irredundant set $S$ for $G=(V, E)$ and any subset $V^{\prime} \subset V$, the set $\left\{v \in S: p n(v, S) \nsubseteq V^{\prime}\right\} \backslash V^{\prime}$ is irredundant in $G\left[V \backslash V^{\prime}\right]$ and has cardinality at least $|S|-\left|V^{\prime}\right|$. (c) The upper irredundance number $\operatorname{IR}(G) \geq \frac{n}{4}$ for any planar graph $G$ of order $n$ (due to the Four-Colour-Theorem). (d) Since every $k$-outerplanar graph has the treewidth at most $3 k-1$, see [20], it follows from Courcelle's Theorem [36] (or [37] for a more algorithmic exposition) that the upper irredundance number on a $k$-outerplanar graph can be found in the time $f(k) n$, where $n$ is the number of vertices in the graph. (e) Then one can show that solving UPPER IRREDUNDANCE exactly on the graph built from $G$ by deleting every ith layer of a planar embedding yields an approximation ratio of $\left(1+\frac{4}{i-1}\right)$ as follows.

1. When deleting every $i$ th layer, by pigeon hole, it is possible to select layers so as not to delete more than $\frac{n}{i}$ vertices.

2. The remaining graph $G^{\prime}$ is a collection of $(i-1)$-outerplanar graphs, and thus can be solved optimally in time $f(i-1) n$, see $(\mathrm{d})$.

3. Let $I R^{\prime}$ be the upper irredundance number for the remaining graph $G^{\prime}$; then $I R^{\prime} \geq n \frac{(i-1)}{4 i}$, see (c) and item 1 .

4. Let $X=I R-I R^{\prime}$; note that $X \leq \frac{n}{i}$.

5. The approximation ratio is then $\frac{I R^{\prime}+X}{I R^{\prime}}=1+\frac{X}{I R^{\prime}} \leq 1+\frac{\frac{n}{i}}{\frac{n(i-1)}{4 i}}=1+\frac{4}{i-1}$.

By a suitable choice of $i$, every desired approximation factor can be achieved. More precisely, for approximation guarantee $1+\varepsilon$, we run the above algorithm for $i=\left\lceil\frac{4}{\varepsilon}\right\rceil+1$. Observations (a) and (b) together guarantee the correctness of the decomposition approach.

In [66], it is shown how to use an approximation scheme for Minimum Domination to solve Nonblocker. The idea can be generalised as follows. Here, by graph parameter we mean the cardinality of a set of vertices satisfying some property. We further assume that we can associate a natural optimisation problem to this parameter. Hence, the discussion below applies in particular to the graph parameters of the (complementary) domination chain.

Proposition 19. Let $\mathcal{G}$ be some graph class and let $\zeta(G)$ be some graph parameter that corresponds to some minimisation problem MIN. Further assume that there exists some constant $c>1$ such that $c \cdot \zeta(G) \leq n(G)$ for all $G \in \mathcal{G}$. If MIN admits a PTAS on $\mathcal{G}$, then also the maximisation problem MAX corresponding to the parameter co- $\zeta(G)$ possesses a PTAS.

Proof. Let $A_{\varepsilon}$ be an algorithm that returns on input $G \in \mathcal{G}, G=(V, E)$ with $n$ vertices, some solution $S_{\varepsilon} \subseteq V$ such that $\left|S_{\varepsilon}\right| \leq(1+\varepsilon) \zeta(G)$. Then, we can design an algorithm $A_{\varepsilon}^{\prime}$ that on input $G \in \mathcal{G}, G=(V, E)$ with $n$ vertices, returns some solution $S_{\varepsilon}^{\prime} \subseteq V$ such that $\left|S_{\varepsilon}^{\prime}\right| \geq(1-\varepsilon)$ co $-\zeta(G)$ as follows.

1. Let $\bar{\varepsilon}=(c-1) \varepsilon$.

2. Let $S_{\bar{\varepsilon}}$ be the solution returned by $A_{\bar{\varepsilon}}$ on input $G$.

3. Return $S_{\varepsilon}^{\prime}:=V \backslash S_{\bar{\varepsilon}}$. 
By definition, $S_{\varepsilon}^{\prime}$ is a feasible solution. The claimed approximation factor is seen as follows:

$$
\begin{aligned}
\left|S_{\varepsilon}^{\prime}\right| & =n(G)-\left|S_{\bar{\varepsilon}}\right| \\
& \geq n(G)-(1+\bar{\varepsilon}) \zeta(G) \\
& =(n(G)-\zeta(G))-\bar{\varepsilon} \zeta(G) \\
& =(n(G)-\zeta(G))-(c-1) \varepsilon \zeta(G) \\
& \geq(n(G)-\zeta(G))-n(G) \varepsilon+\varepsilon \zeta(G) \\
& =(1-\varepsilon)(n(G)-\zeta(G)) \\
& =(1-\varepsilon) \cdot \operatorname{co}-\zeta(G) .
\end{aligned}
$$

This shows the claim.

For instance, if $\mathcal{G}$ is the class of planar graphs without isolated vertices, then $2 \gamma(G) \leq n(G)$ is known, which means that the PTAS for Minimum DominATION on planar graphs transfers to a PTAS for NonBLOCKER, as isolates can be easily dealt with by preprocessing.

A similar transfer of PTAS is also possible from maximisation to minimisation problems. Interestingly enough, in the chain of inequalities we (again) need $c \cdot \zeta(G) \leq n(G)$ for the parameter $\zeta$ now pertaining to the maximisation problem. This is in fact a problem, as usually such a $c>1$ does not exist. Therefore, we refrained from explicitly stating this result here. For instance, although there is a PTAS for UPPER DOMINATION and hence for UPPER IRREDUNDANCE, as sketched above, we see no way how to transfer this to a PTAS for Co-UPPER IRREDUNDANCE. We can only conclude that the known PTAS for Minimum VerTeX Cover can be used to get some $(2+\varepsilon)$-approximation for Co-UPPER IRREDUNDANCE on planar graphs, based on the computations from Theorem 16.

So, unfortunately, we are not aware of a PTAS for any other of the parameters in the (complementary) domination chain. This is particularly true for Lower IRREDUNDANCE and for Minimum INDEPENDENT Domination.

Despite the above improvements on approximability and fixed parameter tractability for the restriction to planarity, it is known that most problems from the domination chain remain NP-hard even when restricted to cubic planar graphs. In fact this is known for all problems except for UPPER and LOWER IRREDUNDANCE; we will show in the following that this hardness also holds for these two problems. These results then also imply that the corresponding complementary versions are NP-hard on planar cubic graphs.

Theorem 20. LOWER IRREDUNDANCE is NP-hard on planar cubic graphs.

Proof. We use the same construction as in [62], where Minimum Domination on planar cubic graphs is reduced to Minimum Independent Domination, as follows: Given a planar cubic graph $G=(V, E)$, construct $G^{\prime}$ from $G$ by replacing every $(u, v) \in E$ by the following planar cubic subgraph with four new vertices $u_{v}, v_{u}, p_{u, v}, q_{u, v}$ :

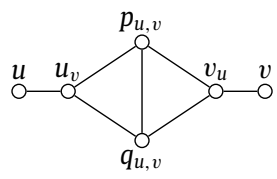

In [62], it is proved that $i\left(G^{\prime}\right)=\gamma(G)+|E|$, which gives us $\operatorname{ir}\left(G^{\prime}\right) \leq \gamma(G)+|E|$ (domination chain). We will now prove that $\operatorname{ir}\left(G^{\prime}\right) \geq \gamma(G)+|E|$, which implies that $\operatorname{ir}\left(G^{\prime}\right)=\gamma(G)+|E|$, that is, $G$ has a dominating set of cardinality at most $k$ if and only if $G^{\prime}$ has a lower irredundant set of cardinality at most $k+|E|$.

Suppose $S$ is a maximal irredundant set for $G^{\prime}$. Consider the sets $D:=S \cap V$ and $V_{u, v}:=S \cap\left\{u_{v}, p_{u, v}, q_{u, v}, v_{u}\right\}$ for all $(u, v) \in E$ and perform the following two modification steps (no changes on $S$ ):

1. For every edge $(u, v)$ in $E$ such that $S \cap\left\{u_{v}, v_{u}, p_{u, v}, q_{u, v}\right\}=\emptyset$, delete $u$ from $D$ and add $u_{v}$ to $V_{u, v}$. Maximality of $S$ implies $u, v \in S$ and $p n(u, S)=\left\{u_{v}\right\}$ and $p n(v, S)=\left\{v_{u}\right\}$. For all edges $(u, w) \in E$ with $w \neq v$, consequently we have $u_{w} \notin p n(u, S)$, which means $S \cap\left\{u_{w}, p_{u, w}, q_{u, w}\right\} \neq \emptyset$. This especially ensures that for all such edges $(u, v)$, one of the vertices $u, v$ remains in $S$.

2. For all $w \in V$ such that $N_{G}[w] \cap D=\emptyset$, we know that $w$ was not deleted by step one, since otherwise its neighbour from the edge which triggered step one would be in $D$. This means $w \notin S$, so by maximality there is a vertex $v \in S \cup\{w\}$ such that $p n(v, S \cup\{w\})=\emptyset$. Since $S$ is irredundant, such a vertex $v$ is either in $\left\{w_{z}, p_{w, z}, q_{w, z}: z \in N_{G}(w)\right\}$ or $v=w$. While there exists a vertex $w \in V$ such that $N_{G}[w] \cap D=\emptyset$, apply modifications according to the following cases for $v$ :

(a) If $v=w_{z}$ for some $z \in N_{G}(w)$, irredundance of $S$ yields $p n\left(w_{z}, S\right) \subseteq\left\{w, w_{z}\right\}$, so especially $q_{w, z} \notin p n\left(w_{z}, S\right)$, so that $\left|V_{w, z}\right|=2$.

Delete $w_{z}$ from $V_{w, z}$ and add $w$ to $D$. 
(b) If $v=q_{w, z}$ for some $z \in N_{G}(w)$ (symmetrically for $p_{w, z}$, irredundance of $S$ yields $p n\left(q_{w, z}, S\right)=\left\{w_{z}\right\}$, which means $z_{w} \in S$. Delete $z_{w}$ from $V_{w, z}$ and add $z$ to $D$.

(c) If $v=w$, especially $w \notin p n(w, S \cap\{w\})$, so there is a vertex $x \in N_{G}(w)$ such that $w_{x} \in S$. If $\left|V_{w, x}\right|=2$, delete $w_{x}$ from $V_{w, x}$ and add $w$ to $D$.

Otherwise, $\left\{x_{w}, p_{x, w}, q_{x, w}\right\} \cap S=\emptyset$, so $x$ was not deleted in step one, so $x \notin S$. Maximality requires $p n\left(v^{\prime}, S \cup\left\{x_{w}\right\}\right)=\emptyset$ for some $v^{\prime} \in S \cup\left\{x_{w}\right\}$. Since $x_{w} \notin N[S]$, this means $v^{\prime}=x_{y}$ for some $y \in N_{G}(x)$ and $p n\left(x_{y}, S\right)=\{x\}$. This implies $S \cap\left\{q_{x, y}, p_{x, y}\right\} \neq \emptyset$, so especially $\left|V_{x, y}\right|=2$.

Delete $x_{y}$ from $V_{x, y}$ and add $x$ to $D$.

Observe that this process of deleting a vertex from some $V_{x, y}$ is not done twice for the same edge $(x, y)$. For every such $V_{x, y}$ we always either delete $x_{y}$ and add $x$ to $D$ or delete $y_{x}$ and add $y$ to $D$. The first two cases (a) and (b) are only considered if neither $x$ nor $y$ is in $D$, so clearly no exchange has happened in a previous step. In the third case, $S$ only contains $x_{y}$ or $y_{x}$, so $V_{x, y}$ will be used at most once to add $x$ or $y$, respectively. After these steps, clearly $D$ is a dominating set for $G$. Since $\left|V_{x, y}\right| \geq 1$ for all $(x, y) \in E$ and $|S|=|D|+\sum_{(x, y) \in E}\left|V_{x, y}\right|$, this dominating set has a cardinality of at most $|S|-|E|$. With the choice of $S$ as a maximal irredundant set for $G^{\prime}$ being arbitrary, we can conclude that $\operatorname{ir}\left(G^{\prime}\right) \geq \gamma(G)+|E|$.

An interesting side note to this proof is that ir, $\gamma$ and $i$ coincide on $G^{\prime}$. Since especially ir and $i$ are known to differ arbitrarily even on cubic graphs [74], this is obviously due to the special structure of $G^{\prime}$. It contains induced $K_{1,3}$ (every original vertex with its neighbourhood), so the result for ir $=\gamma=i$ from [43] does not apply. This makes this construction an interesting candidate to study the characterisation of the graph class for which ir $=i$. With a different construction, we can show the same type of result for UPPER IRREDUNDANCE.

Theorem 21. UPPER IRREDUNDANCE is NP-hard on planar cubic graphs.

Proof. The proof of this result is split into two parts: in (A), we prove NP-hardness on subcubic planar graphs, and then in (B) we show how to modify the construction to get NP-hardness also in the case of cubic graphs.

(A) We reduce from Maximum IndePEndent SET on subcubic planar graphs, proved NP-hard in $[47,49]$. Let $G=(V, E)$ be a subcubic planar input graph for MAXIMUM INDEPENDENT SET. Construct a subcubic planar graph $G^{\prime}$ from $G$ by replacing every edge $(u, v) \in E$ by the following subgraph with the set of six new vertices $V_{u, v}:=\left\{u^{\prime}, u_{1}, u_{2}, v_{1}, v_{2}, v^{\prime}\right\}$.

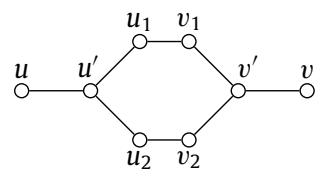

Any maximum independent set $S$ of $G$ can be extended to a maximum independent set in $G^{\prime}$ of cardinality $|S|+3|E|$, since for every edge $(u, v) \in E$ either $u \notin S$ or $v \notin S$, so either $\left\{u^{\prime}, v_{1}, v_{2}\right\}$ or $\left\{v^{\prime}, u_{1}, u_{2}\right\}$ can be added to $S$ without violating independence. This shows that $\alpha\left(G^{\prime}\right) \geq|S|+3|E|$, which implies $\operatorname{IR}\left(G^{\prime}\right) \geq|S|+3|E|$ by the inequalities of the domination chain (Eq. (1)).

Let, on the other hand, $S$ be a maximal irredundant set for $G^{\prime}$. For the induced $C_{6}$ of an edge-gadget, one can easily verify by checking all possibilities that $S$ can contain at most three out of the six vertices in $V_{u, v}$ without violating irredundance. If $\{u, v\} \subset S$ for an edge $(u, v) \in E, S$ can contain at most two vertices from $V_{u, v}$. Consider the sets $S^{\prime}=S \cap V$ and $R=\emptyset$. While there is an edge $(u, v) \in E$ with $\{u, v\} \subset S^{\prime}$, delete $u$ from $S^{\prime}$ and add it to $R$. When this deleting-process ends, $S^{\prime}$ is an independent set in $G$. With $E_{1}:=\{(u, v):\{u, v\} \subset S\}$ and $E_{2}=E-E_{1}$, the cardinality of $S^{\prime}$ can be estimated by:

$$
\left|S^{\prime}\right|=|S|-\sum_{(u, v) \in E}\left|V_{u, v} \cap S\right|-|R| \geq|S|-3\left|E_{2}\right|-2\left|E_{1}\right|-\left|E_{1}\right|=|S|-3|E| .
$$

In conclusion, for any $k \in \mathbb{N}, G$ has an independent set of cardinality at least $k$ if and only if $G^{\prime}$ has an irredundant set of cardinality at least $k+3|E|$. With a planar (sub)cubic input graph $G$, the constructed input graph $G^{\prime}$ is planar and subcubic.

(B) In the previous construction, the resulting graph $G^{\prime}$ is already subcubic and planar and does not contain degree-one vertices. For every vertex of degree two in $G^{\prime}$, add the following subgraph:

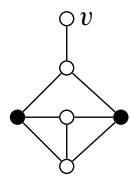


Any choice of two vertices within such a new subgraph dominates all of its new vertices, so any irredundant set for the new graph contains at most two vertices from any of the new subgraphs. If the original vertex $v$ chooses its new neighbour to be a private neighbour in some irredundant set $S$, then $S$ can only contain one of the new vertices of this subgraph. Deleting $v$ from $S$ and choosing, for example, the two black vertices in the above picture to belong to $S$ instead does neither violate irredundance nor change the cardinality of $S$. Let $T$ be the set of degree-two vertices in $G^{\prime}$ and let $G^{\prime \prime}$ denote the graph built from $G^{\prime}$ by adding the above subgraph to every vertex $v \in T$. The graph $G^{\prime \prime}$ is planar, cubic and has an irredundant set of cardinality $k+2|T|$ if and only if $G^{\prime}$ has an irredundant set of cardinality $k$.

Remark 22. As already noted by Manlove [62], the exact reference proving that MAXIMUM InDEPENDENT SET is NP-hard on cubic graphs is not that easy to chase down. The best reference seems to be the constructions from [47,49], but this gives only subcubic (planar) instances. However, with the help of the little gadget from part (B) of the previous proof, any subcubic instance can be easily turned into a cubic one, preserving planarity. Observe that both the full gadget graph and the graph that is obtained by deleting $v$ contain at most two independent vertices.

\section{Graphs of bounded degree}

Bounded degree also improves the fixed parameter tractability and approximability of the problems from the domination chain and their complements. In this section we first present some observations about possible approximation ratios and kernels for graphs of maximum degree $\Delta$. We complement these positive results by L-reductions for UPPER and LOWER IRREDUNDANCE and a scheme of proving approximation-hardness for cubic graphs which, together with previously known results, show that all problems from the domination chain and the complementary domination chain remain APX-hard on cubic graphs.

Observation 23. The approximation-results for UPPER DOMINATION restricted to graphs of bounded degree from [12] are based on Eq. (8) and the fact that every maximal independent set is an upper dominating set which is also true for UPPER IRREDUNDANCE. The approximation by a suitable independent set yields the same approximation-ratio here which especially means that UPPER IRREDUNDANCE can be approximated within factor at most $\frac{6 \Delta^{2}+2 \Delta-3}{10 \Delta}$ for any graph $G$ of bounded degree $\Delta$.

Observation 24. With an algorithmic version of Brooks' Theorem (see for example [69]) one can always find an independent set of cardinality at least $\frac{n}{\Delta}$ for any graph $G$ of bounded degree $\Delta$ in polynomial time. From a parameterised point of view, this immediately gives a $\Delta k$-kernel for MaXimum InDEPENDENT SeT, UpPer Domination and UpPer IRREDUNDANCE for the natural parameter $k$ of these problems, since any bounded-degree graph with more than $\Delta k$ vertices is a trivial "yes"-instance.

Observation 25. Bounded degree $\Delta$ implies $\gamma \geq \frac{n}{\Delta+1}$, which means that any greedy solution yields a $(\Delta+1)$ approximation for Minimal Maximum Independent Set $(i(G)$ in domination chain) and Minimum Domination. For the standard parameterisation this also yields a $(\Delta+1) k$ kernel for these problems since graphs with more than $(\Delta+1) k$ vertices are trivial "no"-instances.

LOWER IRREDUNDANCE is the only problem for which these consequences of bounded degree are less obvious. A more thorough investigation of lower irredundant sets in [35] yields the bound $\operatorname{ir}(G) \geq \frac{2 n}{3 \Delta}$.

Observation 26. The bound from [35] implies that any greedy maximal irredundant set for a graph of bounded degree $\Delta$ is a $1.5 \Delta$-approximation for LoWER IRREDUNDANCE. Parameterised by $k=\operatorname{ir}(G)$, any graph with more than $1.5 \Delta k$ vertices is a trivial "no"-instance which yields a $1.5 \Delta k$ kernel.

Notice that, although the kernel results indicated in the previous two observations look weak at first glance, they allow for lower bound results based on the assumption that $\mathrm{P} \neq \mathrm{NP}$ according to [28].

There is a kind of methodology to link optimisation problems related to the domination chain to those related to the complementary domination chain, which can be stated as follows.

Theorem 27. Assume that the optimisation problem associated to some graph parameter $\zeta$ of the domination chain is APX-hard on cubic graphs. Then, the optimisation problem associated to the complement problem of $\zeta$ is also APX-hard on cubic graphs.

Proof. We claim that the reduction that acts as the identity on graph (instances) and complements solution sets is an L-reduction. Given a cubic graph $G=(V, E)$ of order $n$ with $m=\frac{3}{2} n$ edges as an instance of the optimisation problem belonging to $\zeta$ (and also to the complement problem). Let us distinguish the two optima by writing opt $\mathrm{op}_{\zeta}(G)$ and opt $\mathrm{co}-\zeta_{\zeta}(G)$, respectively. Then, $\mathrm{opt}_{\mathrm{co}-\zeta}(G)=n-\operatorname{opt}_{\zeta}(G)$. Similarly, if $S^{\prime}$ is a solution to $G$ in the complement problem, then $n-\left|S^{\prime}\right|$ is the size of the solution $S:=V \backslash S^{\prime}$ of the original problem. Hence,

$$
\left|\operatorname{opt}_{\zeta}(G)-\right| S||=\left|\left(n-\mathrm{opt}_{\mathrm{co}-\zeta}(G)\right)-\left(n-\left|S^{\prime}\right|\right)\right|=\left|\mathrm{opt}_{\mathrm{co}-\zeta}-\right| S^{\prime}|| .
$$


Moreover, as $\operatorname{ir}(G) \geq \frac{2 n}{9}$ according to [35], which yields opt $t_{\zeta}(G) \geq \frac{2 n}{9}$ by the domination chain,

$$
\operatorname{opt}_{\mathrm{co}-\zeta}(G) \leq n \leq \frac{9}{2} \operatorname{opt}_{\zeta}(G),
$$

which proves the claim.

As a matter of fact, Cockayne and Mynhardt [35] provide a lower bound on $\operatorname{ir}(G)$ for any graph of maximum degree $\Delta$. Hence, the reasoning of the preceding proof allows to transfer APX-hardness results for all graph classes with bounded maximum degree $\Delta$ from a domination chain parameter to the complementary parameter, not only for (sub)cubic graphs.

Theorem 3.3 in [3] shows that Minimum DominATION, restricted to cubic graphs, is APX-hard. We can use Theorem 27 to immediately deduce:

Corollary 28. The complement problem corresponding to MinimuM DoMINATION is APX-hard when restricted to cubic graphs.

This sharpens earlier results [15] that only considered the subcubic case.

Corollary 29. LOWER IRREDUNDANCE restricted to cubic graphs is APX-hard. Similarly, Co-LOWER IRREDUNDANCE is APX-hard on cubic graphs.

Proof. The reduction from Theorem 20 can be seen as an L-reduction from the APX-hard MinIMUM DominATION problem on cubic graphs [3] to LowER IRREDUNDANCE on cubic graphs. Observe that $\gamma(G) \geq \frac{n}{4}$ and $|E|=\frac{3}{2} n$ for any cubic graph $G$, which gives $\operatorname{ir}\left(G^{\prime}\right)=\gamma(G)+|E| \leq 7 \gamma(G)$. Furthermore, any maximal irredundant set of cardinality val' for $G^{\prime}$ can be used to compute a dominating set for $G$ of cardinality $v a l=v a l^{\prime}-|E|$, which yields $v a l-\gamma(G)=v a l^{\prime}-\operatorname{ir}\left(G^{\prime}\right)$. Together with Theorem 27 the result for Co-LOWER IRREDUNDANCE follows.

The computations in the previous proof can be carried out completely analogously for UPPER IRREDUNDANCE and CO-UPPER IRREDUNDANCE.

Corollary 30. UPPER IRREDUNDANCE is APX-hard on cubic graphs. Similarly, Co-UPPER IRREDUNDANCE is APX-hard on cubic graphs.

Proof. The reductions from Theorem 21 can be seen as L-reductions. Since the composition of L-reductions is an L-reduction, we obtain APX-hardness of UPPER IRREDUNDANCE on cubic graphs from the APX-hardness of MAXIMUM INDEPENDENT SET on cubic graphs. Observe that $\alpha(G) \geq \frac{n}{4}$ and $|E|=\frac{3}{2} n$ for any cubic graph $G$, which gives IR $\left(G^{\prime}\right)=$ $\alpha(G)+3|E| \leq 19 \alpha(G)$. Furthermore, any maximal irredundant set of cardinality val for $G^{\prime}$ can be used to compute a dominating set for $G$ of cardinality $v a l=v a l^{\prime}-3|E|$, which yields $v a l-\alpha(G)=v a l^{\prime}-\operatorname{IR}\left(G^{\prime}\right)$. $\operatorname{Moreover}, \operatorname{IR}\left(G^{\prime \prime}\right)=\operatorname{IR}\left(G^{\prime}\right)+8 \mid E$ and $\operatorname{IR}\left(G^{\prime}\right) \geq \frac{19 n}{4}$, gives $\operatorname{IR}\left(G^{\prime \prime}\right) \leq \frac{67 \operatorname{IR}\left(G^{\prime}\right)}{19}$. Furthermore, any maximal irredundant set of cardinality $v a l^{\prime \prime}$ for $G^{\prime \prime}$ can be used to compute a maximal irredundant set for $G^{\prime}$ of cardinality $v a l^{\prime}=v a l^{\prime \prime}-8|E|$, which yields $v a l^{\prime}-\operatorname{IR}\left(G^{\prime}\right)=v a l^{\prime \prime}-\operatorname{IR}\left(G^{\prime \prime}\right)$. Together with Theorem 27, the result for Co-UPPER IRREDUNDANCE follows.

Another problem in the domination chain that was somewhat neglected so far with respect to approximation in cubic graphs is Minimum Independent Domination. David Manlove has obtained an NP-hardness result for Minimum INDEPENDENT DOMINATION on cubic planar graphs [62]. By making use of this reduction, which turns out to be an L-reduction and is very much the same as the one we showed in Theorem 20 , we can conclude:

Corollary 31. Minimum MaXimal IndePEndence is APX-hard on cubic graphs.

Notice that the construction provided in the proof of [56], Theorem A.2, only shows APX-hardness for graphs of maximum degree four, or any other bigger maximum degree. Likewise, this is true for the complement problem, also known as Maximum Minimal Vertex Cover, due to Theorem 27.

Corollary 32. Maximum Minimal Vertex Cover is APX-hard on cubic graphs.

This improves on earlier results for MAXimum Minimal VerTex Cover, for instance, the APX-hardness shown in [64] for graphs of maximum degree bounded by five.

\section{Everywhere dense graphs}

In [7], Arora et al. presented a unified framework for proving polynomial time approximation schemes for (average) dense graphs, mainly for MAX Cut type problems, and for Min BISECTION for everywhere dense graphs. Concerning the problems from the domination chain Minimum VerTex Cover and Minimum Domination were studied; in [31], Minimum VERTEX COVER is proved APX-hard on everywhere dense graphs and in [50], it is proved that MinimuM DominATION 
is NP-hard on (average) dense graphs. We will show inapproximation results for more domination-chain problems on everywhere dense graphs. Interestingly, we can make use of our reductions for sparse (cubic) graphs:

Theorem 33. For any $\varepsilon>0$, Co-UPPER IRREDUNDANCE is APX-hard and UPPER IRREDUNDANCE is not $n^{1-\delta}$-approximable for any $\delta>0$, if $\mathrm{P} \neq \mathrm{NP}$, for everywhere- $\varepsilon$-dense graphs.

Proof. We construct an E-reduction from UPPER IRREDUNDANCE to UPPER IRREDUNDANCE on everywhere- $\varepsilon$-dense graphs. Given a connected graph $G=(V, E)$ of order $n$, we construct a dense graph $G^{\prime}$ by joining a clique $C$ of $\left\lceil\frac{\varepsilon n}{1-\varepsilon}\right\rceil$ new vertices to $G$. Any vertex $v \in V$ has at least $\left\lceil\frac{\varepsilon n}{1-\varepsilon}\right\rceil \geq \varepsilon\left\lceil\frac{n}{1-\varepsilon}\right\rceil$ many neighbours in $G^{\prime}$ and any vertex in the added clique has an even higher degree if $n \geq 4$. The minimum degree of $G^{\prime}$ is hence at least $\varepsilon n^{\prime}$, where $n^{\prime}=n+\left\lceil\frac{\varepsilon n}{1-\varepsilon}\right\rceil=\left\lceil\frac{n}{1-\varepsilon}\right\rceil$ is the number of vertices of $G^{\prime}$. As any maximal irredundant set of $G^{\prime}$ that contains a vertex of $C$ is a singleton $\operatorname{set}, \operatorname{opt}\left(G^{\prime}\right)=\operatorname{opt}(G)$ and, w.l.o.g., any maximum irredundant set in $G^{\prime}$ is a subset of $V$ which makes it a maximal irredundant set of $G$. Since by Theorem 14 UPPER IRREDUNDANCE on general graphs is not $n^{1-\delta}$-approximable for any $\delta>0$, if $\mathrm{P} \neq \mathrm{NP}$, this reduction yields the same inapproximability for the restriction to everywhere- $\varepsilon$-dense graphs.

For Co-UPPER IRREDUNDANCE we reduce from Co-UPPER IRREDUNDANCE on cubic graphs and use the same construction for $G^{\prime}$ which yields $\operatorname{opt}\left(G^{\prime}\right)=\operatorname{opt}(G)+\left\lceil\frac{\varepsilon n}{1-\varepsilon}\right\rceil$. Given any solution $S^{\prime}$ in $G^{\prime}$, we can transform it into a new one containing all new vertices and some vertices from $V$. The set $S^{\prime} \cap V$ is a solution for $G$. In a cubic graph, the optimum value of the complement of an upper irredundant set is at least $n / 4$ using inequality (9) and the fact that $\tau(G) \geq n / 2$ (as $G$ is connected and non-trivial) and thus $\operatorname{opt}(G) \geq n / 4$. Thus:

$$
\operatorname{opt}\left(G^{\prime}\right) \leq \operatorname{opt}(G)+\left\lceil\frac{\varepsilon n}{1-\varepsilon}\right\rceil \leq \operatorname{opt}(G)+\left\lceil\frac{4 \varepsilon \operatorname{opt}(G)}{1-\varepsilon}\right\rceil \leq\left\lceil\frac{1+4 \varepsilon}{1-\varepsilon}\right\rceil \operatorname{opt}(G),
$$

which makes this reduction an L-reduction from Co-UpPeR IRREDUNDANCE to Co-UpPeR IRREDUNDANCE on cubic graphs and hence transfers the APX-hardness from Corollary 30 to Co-UPPER IRREDUNDANCE restricted to everywhere- $\varepsilon$-dense graphs.

Observe that the arguments and the computations of the previous proof are also valid for (CO-)UPPER DoMINATION, since Co-UPPER Domination is also APX-hard on cubic graphs [12] and UPPER DominATION is not $n^{1-\delta}$-approximable for any $\delta>0$ on general graphs [12] (if $\mathrm{P} \neq \mathrm{NP}$ ), so that we can conclude a similar result.

Corollary 34. For any $\varepsilon>0$, Co-UPPER DominATION is APX-hard and UPPER DominATION is not $n^{1-\delta}$-approximable for any $\delta>0$, if $\mathrm{P} \neq \mathrm{NP}$, for everywhere- $\varepsilon$-dense graphs.

The inapproximability result from [72] with the above reduction yields:

Proposition 35. For any $\varepsilon>0$, Maximum IndePendent Set is not $n^{1-\delta}$-approximable for any $\delta>0$, if $\mathrm{P} \neq \mathrm{NP}$, for everywhere- $\varepsilon$-dense graphs. ${ }^{2}$

Theorem 36. For any $\varepsilon>0$, Maximum Minimal Vertex Cover is APX-hard and Minimum Maximal Independent Set is not $n^{1-\delta}$-approximable for any $\delta>0$, if $P \neq N P$, for everywhere- $\varepsilon$-dense graphs.

Proof. We give an E-reduction from Minimum Maximal Independent Set on general graphs to Minimum Maximal INDEPENDENT SET on everywhere- $\varepsilon$-dense graphs. Consider for a graph $G$ the family $\left\{G^{j}: j \in \mathbb{N}\right\}$, recursively defined by $G^{0}:=G$ and $G^{j+1}:=G^{j}+G^{j}$ (where "+" denotes graph join ${ }^{3}$ ). If the order of $G$ is $n$, the order of $G^{j}$ is $2^{j} n$ for every $j \in \mathbb{N}$. Also every $v \in G^{j}$ has degree at least $n\left(2^{j}-1\right)$ which means that $G^{j}$ is $\left(1-1 / 2^{j}\right)$-dense. Let $V$ be the vertices of $G$ and $V \cup V^{\prime}$ be the vertices of $G+G$. For any independent set $S$ of $G+G$ either $S \subseteq V$ or $S \subseteq V^{\prime}$, which means that independent sets in $G+G$ always yield equivalent independent sets in $G$ and hence $i(G)=i(G+G)$. Inductively, this argument implies $i(G)=i\left(G^{j}\right)$ for all $j \in \mathbb{N}$. For $j=\left\lceil\log _{2}(1 /(1-\varepsilon))\right\rceil$, the graph $G^{j}$ hence yields the aforementioned E-reduction since any independent set in $G^{j}$ yields an independent set in $G$ of the same size.

Starting with a cubic graph $G, G^{j}$ yields an L-reduction from Maximum Minimal Vertex Cover on cubic graphs, which is APX-hard by Corollary 32, to MAXImum Minimal VerTex Cover on everywhere- $\varepsilon$-dense graphs, since for cubic graphs co $-i(G) \geq \frac{n}{2}$ and hence co $-i\left(G^{j}\right)<2^{j} n \leq 2^{j+1}$ co $-i(G)$.

Remark 37. The reductions from this section are especially polynomial time reductions which preserve the optimal value which makes them FPT-reductions. As a consequence, Minimum InDEPENDENT Domination remains W[2]-hard

2 We were informed about this fact by Marek Karpiński.

3 For two graphs $G=(V, E)$ and $G^{\prime}=\left(V^{\prime}, E^{\prime}\right)$ with $V \cap V^{\prime}=\emptyset$, their join is defined as $G+G^{\prime}=\left(V \cup V^{\prime}, E \cup E^{\prime}\right)$. 
and MAXimum Independent Set, Upper Domination and UpPeR IRREDUndAnce remain W[1]-hard on everywhere- $\varepsilon$-dense graphs.

\section{A special flavour of minimax/maximin problems}

Half of the parameters in the domination chain can be defined as either, in case of minimax problems, looking for the smallest of all (inclusion-wise) maximal vertex sets with a certain property $(i(G)$ is the size of the smallest maximal independent set; similarly, $\operatorname{ir}(G)$ is defined), or, in case of maximin problems, looking for the largest of all minimal vertex sets with a certain property $(\Gamma(G)$ is an example). Also, the complementary problems share this flavour; for instance, co $-i(G)$ can be seen as looking for the largest of all minimal vertex covers. Further discussions can be found in [61].

Typical exact algorithms for maximisation problems fix certain subsets to be part of the solution. In the decision variant, when a parameter value that lower-bounds the size of the solution is part of the input, we might have a sufficient number of vertices in our partial solution and now want to (rather immediately) announce that a sufficiently large solution exists. This is not a problem for determining $\alpha(G)$ or $\operatorname{IR}(G)$, but this may become problematic in the case of maximin problems. In the following we consider the extension-problem for the other two maximin problems related to the domination-chain: co $-i(G)$ and $\mathrm{co}-\operatorname{ir}(G)$. The problem of computing a solution for $\mathrm{co}-i(G)$ which contains a certain given subset of vertices can formally be stated as follows:

Minimal Vertex Cover Extension

Input: A graph $G=(V, E)$, a set $S \subseteq V$.

Question: Does $G$ possess a minimal vertex cover $S^{\prime}$ with $S^{\prime} \supseteq S$ ?

Observe that this extension problem can also be seen as a kind of subset problem for independent sets by rephrasing the question to: Is there a maximal independent set $S^{\prime}$ for $G$ with $S^{\prime} \subseteq V-S$ ? In more general terms, one can view the extension-version of some maximin problem as exclusion-version of the complementary minimax problem.

Theorem 38. Minimal Vertex COVER EXTENSION is NP-hard even restricted to planar cubic graphs.

Proof. Consider the following simple reduction from Satisfiability: For a formula $c_{1} \wedge \cdots \wedge c_{m}$ over variables $x_{1}, \ldots, x_{n}$, let $G=(V, E)$ be the graph with vertices $v_{i}, \bar{v}_{i}$ for every $i=1, \ldots, n$ and $c_{1}, \ldots, c_{m}$ and edges connecting every clause with its literals and connecting $v_{i}$ with $\bar{v}_{i}$ for every $i$. For this graph, the set $S=\left\{c_{1}, \ldots, c_{m}\right\}$ can be extended to a minimal vertex cover if and only if the formula $c_{1} \wedge \cdots \wedge c_{m}$ is satisfiable.

If there exists an assignment $\phi$ for $x_{1}, \ldots, x_{n}$ which satisfies $c_{1} \wedge \cdots \wedge c_{m}$, the set $S^{\prime}:=S \cup\left\{v_{i}: \phi\left(x_{i}\right)=0\right\} \cup\left\{\bar{v}_{i}: \phi\left(x_{i}\right)=1\right\}$ is a minimal vertex cover for $G$. Since for each edge $\left(v_{i}, \bar{v}_{i}\right)$ either $v_{i} \in S^{\prime}$ or $\bar{v}_{i} \in S^{\prime}$ and all vertices $c_{j}$ are in $S^{\prime}$, every edge is covered. Every vertex $v_{i} \in S^{\prime}$ (or $\bar{v}_{i} \in S^{\prime}$ ) uniquely covers the edge $\left(v_{i}, \bar{v}_{i}\right.$ ), so $S^{\prime}-\left\{v_{i}\right\}$ (or $S^{\prime}-\left\{\bar{v}_{i}\right\}$ ) is not a vertex cover. Since $\phi$ is satisfying for $c_{1} \wedge \cdots \wedge c_{m}$, every clause $c_{j}$ has at least one literal which is not in $S^{\prime}$. This means that the edge corresponding to this satisfying literal is uniquely covered by $c_{j} \in S^{\prime}$ or, in other words, that $S^{\prime}-\left\{c_{j}\right\}$ is not a vertex cover, so $S^{\prime}$ is minimal.

Let, on the other hand, $S^{\prime}$ be a minimal vertex cover for $G$ which extends $S$. Every edge $\left(v_{i}, \bar{v}_{i}\right)$ has to be covered by $S^{\prime}$ which means that $S^{\prime} \cap\left\{v_{i}, \bar{v}_{i}\right\} \neq \emptyset$. The setting $\phi\left(x_{i}\right)=0$ if $\bar{v}_{i} \notin S^{\prime}$ and $\phi\left(x_{i}\right)=1$ if $v_{i} \notin S^{\prime}$ is hence not contradictory. Minimality requires that there is an edge $\left(c_{j}, x\right)$ for which $x \notin S^{\prime}$ for each $j=1, \ldots, m$. The only possible vertices $x$ for these private edges are literals of the clause $c_{j}$ which means that there is either a $v_{i} \notin S^{\prime}$ or a $\bar{v}_{i} \notin S^{\prime}$ which is literal in $c_{j}$. In terms of SATISFIABILITY, this implies that the assignment $\phi$ can be extended to a satisfying assignment for $c_{1} \wedge \cdots \wedge c_{m}$.

To prove hardness for the restriction to planar cubic graphs, consider reducing not from general SATISFIABILITY but from the NP-hard 4-Bounded PLANAR 3-ConNeCTED SAT (4P3C3SAT) [59]. For a 4P3C3SAT-formula $c_{1} \wedge \cdots \wedge c_{m}$, the associated graph $G=(V, E)$ with vertex-set $\left\{c_{1}, \ldots, c_{m}\right\} \cup\left\{x_{1}, \ldots, x_{n}\right\}$ and edges connecting each clause to the three variables which occur in it is planar and the vertices $x_{i}$ have degree at most four. Fix some planar embedding of $G$ and let $c_{1}^{i}, c_{2}^{i}, c_{3}^{i}, c_{4}^{i}$ be the (possibly not existing) clauses in which $x_{i}$ appears, arranged in the chosen planar embedding in clockwise order:

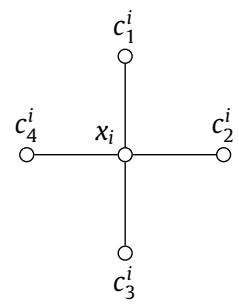


Create the graph $G^{\prime}$ from $G$ by replacing each $x_{i}$ according to the following cases:

(a) If the variable $x_{i}$ appears positively in clauses $c_{1}^{i}, c_{2}^{i}, c_{3}^{i}$ and negated in $c_{4}^{i}$, replace $x_{i}$ by:

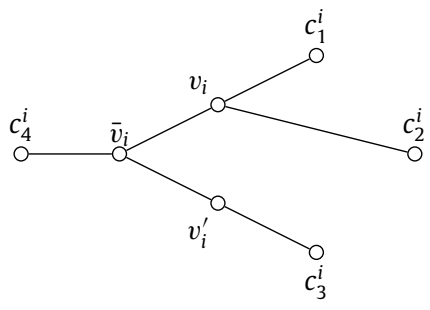

(b) If the variable $x_{i}$ appears positively in clauses $c_{1}^{i}, c_{2}^{i}$ and negated in $c_{3}^{i}, c_{4}^{i}$, replace $x_{i}$ by:

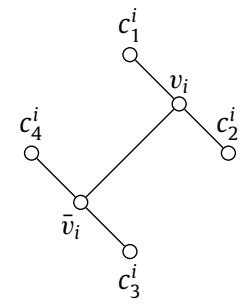

(c) If the variable $x_{i}$ appears positively in clauses $c_{1}^{i}, c_{3}^{i}$ and negated in $c_{2}^{i}, c_{4}^{i}$, replace $x_{i}$ by:

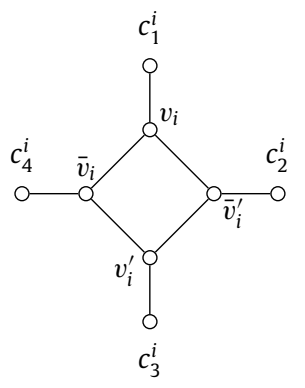

All other cases are rotations of the above three cases and/or invert the roles of $v_{i}$ and $\bar{v}_{i}$ and $v_{i}^{\prime}$ and $\bar{v}_{i}^{\prime}$.

The same argument as above for general SATISFIABILITY, the set $S=\left\{c_{1}, \ldots, c_{m}\right\}$ can be extended to a minimal vertex cover for $G^{\prime}$ if and only if the formula $c_{1} \wedge \cdots \wedge c_{m}$ is satisfiable. To see this, observe that in the adjusted construction $v_{i}^{\prime}$ is copy of $v_{i}$ and $\bar{v}_{i}^{\prime}$ as copy of $\bar{v}_{i}$ in the sense that the edges among the vertices $\left\{v_{i}, v_{i}^{\prime}, \bar{v}_{i}, \bar{v}_{i}^{\prime}\right\}$ force that either the set $\left\{v_{i}, v_{i}^{\prime}\right\}$ (corresponding to $\phi\left(x_{i}\right)=0$ ) or the set $\left\{\bar{v}_{i}, \bar{v}_{i}^{\prime}\right\}$ (corresponding to $\phi\left(x_{i}\right)=1$ ) is included in a vertex cover for $G^{\prime}$ and conversely suffices to cover all edges in case of a satisfying assignment $\phi$.

$G^{\prime}$ is planar and subcubic. To construct a planar cubic instance add the following subgraph to every vertex $v$ of degree two and put the black vertices to $S$ :

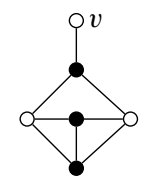

All new edges introduced by these additional subgraphs are already covered by the vertices added to $S$ and these new vertices in $S$ obviously do not cover any original edge. These additional subgraphs consequently do not affect the possibility to turn $S$ into a minimal vertex cover for $G^{\prime}$.

The maximin problem co $-\operatorname{ir}(G)$ can also be considered with respect to extension. Since complements of irredundant sets are rather tricky, we describe this problem in terms of the complementary problem $\operatorname{ir}(G)$ :

Minimal Co-IRREdundant EXTENSION

Input: A graph $G=(V, E)$, a set $S \subseteq V$.

Question: Does $G$ possess a maximal irredundant set $S^{\prime}$ with $S^{\prime} \subseteq V-S$ ?

Theorem 39. Minimal Co-IrRedundant Extension is NP-hard. 
Proof. Just like for Minimal Vertex Cover Extension, we reduce from Satisfiability. Given a formula $c_{1} \wedge \cdots \wedge c_{m}$ over variables $x_{1}, \ldots, x_{n}$, construct a graph $G=(V, E)$ with vertices $\left\{k_{1}, \ldots, k_{m}\right\} \cup\left\{o_{i}, p_{i}, f_{i}^{1}, f_{i}^{2}, u_{i}, \bar{u}_{i}, v_{i}, \bar{v}_{i}: i=1, \ldots, n\right\}$ and edges connecting $k_{j}$ to $v_{i}$ or $\bar{v}_{i}$ if $x_{i}$ or $\bar{x}_{i}$ is literal in $c_{j}$ respectively. Further, the vertices $\left\{o_{i}, p_{i}, f_{i}^{1}, f_{i}^{2}, u_{i}, \bar{u}_{i}, v_{i}, \bar{v}_{i}: 1 \leq i \leq n\right\}$ induce $n$ non-connected subgraphs of the following structure:

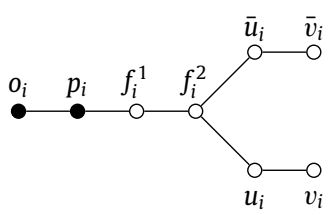

With this construction, $c_{1} \wedge \cdots \wedge c_{m}$ is satisfiable if and only if there is an irredundant set for $G$ which does not contain any vertex from $S:=\left\{k_{1}, \ldots, k_{m}\right\} \cup\left\{o_{i}, p_{i}: 1 \leq i \leq m\right\}$.

Suppose $c_{1} \wedge \cdots \wedge c_{m}$ is satisfiable by some assignment $\phi$. We claim that $S^{\prime}:=\left\{u_{i}: \phi\left(x_{i}\right)=1\right\} \cup\left\{\bar{u}_{i}: \phi\left(x_{i}\right)=\right.$ $0\} \cup\left\{f_{i}^{1}, f_{i}^{2}: i=1, \ldots, n\right\}$ is a maximal irredundant set in $G$. The private neighbourhoods with respect to $S^{\prime}$ are: $p n\left(f_{i}^{1}, S^{\prime}\right)=\left\{p_{i}\right\}, p n\left(f_{i}^{2}, S^{\prime}\right)=\left\{u_{i}, \bar{u}_{i}\right\}-S^{\prime}, p n\left(u_{i}, S^{\prime}\right)=\left\{v_{i}\right\}, p n\left(\bar{u}_{i}, S^{\prime}\right)=\left\{\bar{v}_{i}\right\}$, all of which are non-empty which means that $S^{\prime}$ is irredundant. About maximality, adding a vertex $v$ from $V-S^{\prime}$ to $S^{\prime}$ means one of the following cases:

1. $v=o_{i}$ or $v=p_{i}$ for some $i$ yields $p n\left(f_{i}^{1}, S^{\prime} \cup\{v\}\right)=\emptyset$.

2. $v=\bar{u}_{i}$ or $v=\bar{v}_{i}$ for some $i$ with $\phi\left(x_{i}\right)=1$ yields $p n\left(f_{i}^{2}, S^{\prime} \cup\{v\}\right)=\emptyset$.

3. $v=u_{i}$ or $v=v_{i}$ for some $i$ with $\phi\left(x_{i}\right)=0$ yields $p n\left(f_{i}^{2}, S^{\prime} \cup\{v\}\right)=\emptyset$.

4. $v=v_{i}$ for some $i$ with $\phi\left(x_{i}\right)=1$ yields $p n\left(u_{i}, S^{\prime} \cup\{v\}\right)=\emptyset$.

5. $v=\bar{v}_{i}$ for some $i$ with $\phi\left(x_{i}\right)=0$ yields $p n\left(\bar{u}_{i}, S^{\prime} \cup\{v\}\right)=\emptyset$.

6. $v=k_{j}$ for some $j$ implies $p n\left(u_{i}, S^{\prime} \cup\{v\}\right)=\emptyset$ (or $p n\left(\bar{u}_{i}, S^{\prime} \cup\{v\}\right)=\emptyset$ ) for each $i$ with $x_{i}$ (or $\bar{x}_{j}$ ) literal in $c_{j}$. Since $\phi$ is a satisfying assignment for $c_{1} \wedge \cdots \wedge c_{m}, \phi$ satisfies at least one literal of each $c_{j}$ and the corresponding $u_{i}$ (or $\bar{u}_{i}$ ) is consequently in $S^{\prime}$.

Overall, any choice of $v \in V-S^{\prime}$ yields some $w \in S^{\prime}$ such that $p n\left(w, S^{\prime} \cup\{v\}\right)=\emptyset$ which proves maximality of $S^{\prime}$.

Let $S^{\prime}$ be a maximal irredundant set for $G$ with $S^{\prime} \cap S=\emptyset$. Maximality of $S^{\prime}$ requires at least one vertex $w$ for which $p n\left(w, S^{\prime} \cup\left\{o_{i}\right\}\right)=\emptyset$ for each $i=1, \ldots, n$. For any set which does not contain $o_{i}$ or $p_{i}$, the only possible choice for such a vertex $w$ is $f_{i}^{1}$. $p n\left(f_{i}^{1}, S^{\prime} \cup\left\{o_{i}\right\}\right)=\emptyset$ especially requires that $f_{i}^{1}$ has at least one neighbour in $S^{\prime} \cup\left\{o_{i}\right\}$ which means $f_{i}^{2} \in S^{\prime}$ for all $i=1, \ldots, n$. Irredundance of $S^{\prime}$ requires at least one private neighbour for $f_{i}^{2}$, which means that either $S^{\prime} \cap\left\{u_{i}, v_{i}\right\}=\emptyset$ or $S^{\prime} \cap\left\{\bar{u}_{i}, \bar{v}_{i}\right\}=\emptyset$. This allows to define the (partial) assignment:

$$
\phi\left(x_{i}\right)= \begin{cases}1 & \text { if } S^{\prime} \cap\left\{u_{i}, v_{i}\right\} \neq \emptyset \\ 0 & \text { if } S^{\prime} \cap\left\{\bar{u}_{i}, \bar{v}_{i}\right\} \neq \emptyset\end{cases}
$$

Suppose there is a clause $c_{j}$ which is not satisfied by this assignment. This means that for all neighbours $v_{i}$ (or $\bar{v}_{i}$ ) of $k_{j}$, $S^{\prime} \cap\left\{v_{i}, u_{i}\right\}=\emptyset$ (or $S^{\prime} \cap\left\{\bar{v}_{i}, \bar{u}_{i}\right\}=\emptyset$ ). This however means that $p n\left(k_{j}, S^{\prime} \cup\left\{k_{j}\right\}\right) \supset\left\{k_{j}\right\}$ and further, $k_{j}$ can only affect private neighbourhoods from vertices in $\left\{u_{i}, v_{i}\right\}$ (or $\left\{\bar{u}_{i}, \bar{v}_{i}\right\}$ ) for indices $i$ such that $x_{i}$ (or $\bar{x}_{i}$ ) is literal in $c_{j}$, none of which are in $S^{\prime}$. In other words, if $c_{j}$ is not satisfied by $\phi, S^{\prime} \cup\left\{k_{j}\right\}$ is irredundant, a contradiction to the maximality of $S^{\prime}$.

\section{Summary, open problems and prospects}

We have presented a survey of the complexity landscape of the domination chain. As can be seen from our tables, the status of most combinatorial problems has now been solved. However, there are still several question marks in these tables, and also the positive (algorithmic) results implicitly always ask for possible improvements. To make one question from [6] explicit in this context: Do there exist subexponential-time approximation algorithms that improve on the factor-2 approximation bound for VERTEX COVER under UGC? Even more intriguingly, only surprisingly few PTAS are available. It seems to us as if general results as given in [46] are not applicable here. Also (not expressed as a question mark in one of the tables) the question whether UPPER DominATION is in W[1] or hard for W[2] is still open.

It is always good to have a rather systematic treatment of matters, as this helps to understand the nature of these subjects better. For the investigation of complexity aspects of graph parameters, chains of inequalities like the domination chain not only help unify proofs, but also to find spots that have not been investigated yet. Also, the idea of looking at the complementary chain should work out in each case.

As an example of a similar chain of parameters, we only mention the Roman domination chain [26]. Our knowledge on the complexity of these parameters is still very underdeveloped; most of what we know is concerning Roman domination and its complementary version, which is also called the differential of a graph; see [1,15-17]. 


\section{Acknowledgements}

We gratefully acknowledge the support by the Deutsche Forschungsgemeinschaft, Germany, grant FE 560/6-1, which funded the PhD position for Katrin Casel and Mercator Felowship for Ljiljana Brankovic. Additionally, Ljiljana Brankovic and Henning Fernau thank the Paris Dauphine University, France for supporting research visits and collaboration with Cristina Bazgan.

\section{References}

[1] F.N. Abu-Khzam, C. Bazgan, M. Chopin, H. Fernau, Data reductions and combinatorial bounds for improved approximation algorithms, J. Comput System Sci. 82 (3) (2016) 503-520.

[2] J. Alber, H.L. Bodlaender, H. Fernau, T. Kloks, R. Niedermeier, Fixed parameter algorithms for DominATING SET and related problems on planar graphs, Algorithmica 33 (2002) 461-493.

[3] P. Alimonti, V. Kann, Some APX-completeness results for cubic graphs, Theoret. Comput. Sci. 237 (1-2) (2000) 123-134.

[4] R.B. Allan, R. Laskar, On domination and independent domination numbers of a graph, Discrete Math. 23 (2) (1978) 73-76.

[5] R.B. Allan, R. Laskar, S. Hedetniemi, A note on total domination, Discrete Math. 49 (1) (1984) 7-13.

[6] S. Arora, B. Barak, D. Steurer, Subexponential algorithms for unique games and related problems, J. ACM 62 (5) (2015) 42.

[7] S. Arora, D.R. Karger, M. Karpinski, Polynomial time approximation schemes for dense instances of NP-hard problems, J. Comput. System Sci. 58 (1) (1999) 193-210.

[8] S. Athanassopoulos, I. Caragiannis, C. Kaklamanis, Analysis of approximation algorithms for $k$-set cover using factor-revealing linear programs, Theory Comput. Syst. 45 (3) (2009) 555-576.

[9] S. Athanassopoulos, I. Caragiannis, C. Kaklamanis, M. Kyropoulou, An improved approximation bound for spanning star forest and color saving, in: R. Královic, D. Niwinski (Eds.), Mathematical Foundations of Computer Science 2009, 34th International Symposium, MFCS, in: LNCS, vol. 5734, Springer, 2009, pp. 90-101.

[10] G. Ausiello, P. Creczenzi, G. Gambosi, V. Kann, A. Marchetti-Spaccamela, M. Protasi, Complexity and Approximation; Combinatorial Optimization Problems and their Approximability Properties, Springer, 1999.

[11] B. Baker, Approximation algorithms for NP-complete problems on planar graphs, J. ACM 41 (1994) 153-180.

[12] C. Bazgan, L. Brankovic, K. Casel, H. Fernau, K. Jansen, M. Lampis, M. Liedloff, J. Monnot, V. Paschos, The many facets of upper domination, Theoret. Comput. Sci. 717 (2018) 2-25.

[13] C. Berge, Graphs and Hypergraphs, North-Holland, Amsterdam, 1973.

[14] P. Berman, T. Fujito, On the approximation properties of independent set problem in degree 3 graphs, in: S.G. Akl, F.K.H.A. Dehne, J.-R. Sack, N. Santoro (Eds.), Algorithms and Data Structures, 4th International Workshop, WADS, in: LNCS, vol. 955, Springer, 1995, pp. 449-460.

[15] S. Bermudo, H. Fernau, Computing the differential of a graph: hardness, approximability and exact algorithms, Discrete Appl. Math. 165 (2014) 69-82.

[16] S. Bermudo, H. Fernau, Combinatorics for smaller kernels: The differential of a graph, Theoret. Comput. Sci. 562 (2015) 330-345.

[17] S. Bermudo, H. Fernau, J.M. Sigarreta, The differential and the Roman domination number of a graph, Appl. Anal. Discrete Math. 8 (2014) $155-171$.

[18] D. Bienstock, C.L. Monma, On the complexity of embedding planar graphs to minimize certain distance measures, Algorithmica 5 (1990) 93-109.

[19] D. Binkele-Raible, L. Brankovic, M. Cygan, H. Fernau, J. Kneis, D. Kratsch, A. Langer, M. Liedloff, M. Pilipczuk, P. Rossmanith, J.O. Wojtaszczyk, Breaking the $2^{n}$-barrier for IRREDUNDANCE: Two lines of attack, J. Discrete Algorithms 9 (2011) 214-230.

[20] H.L. Bodlaender, A partial k-arboretum of graphs with bounded treewidth, Theoret. Comput. Sci. 209 (1998) 1-45.

[21] B. Bollobás, E.J. Cockayne, Graph-theoretic parameters concerning domination, independence, and irredundance, J. Graph Theory 3 (1979) $241-249$.

[22] N. Boria, F.D. Croce, V.T. Paschos, On the max min vertex cover problem, Discrete Appl. Math. 196 (2015) 62-71.

[23] N. Bourgeois, F.D. Croce, B. Escoffier, V.T. Paschos, Fast algorithms for min independent dominating set, Discrete Appl. Math. 161 (4-5) (2013) $558-572$

[24] L. Brankovic, H. Fernau, A novel parameterised approximation algorithm for minimum VerTex cover, Theoret. Comput. Sci. 511 (2013) 85-108.

[25] P. Chalermsook, B. Laekhanukit, D. Nanongkai, Graph products revisited: Tight approximation hardness of induced matching, poset dimension and more, in: Proceedings of the Twenty-Fourth Annual ACM-SIAM Symposium on Discrete Algorithms, SODA, 2013, pp. 1557-1576.

[26] M. Chellali, T.W. Haynes, S.M. Hedetniemi, S.T. Hedetniemi, A.A. McRae, A roman domination chain, Graphs Combin. 32 (1) (2016) 79-92.

[27] J. Chen, Vertex cover kernelization, in: M. Kao (Ed.), Encyclopedia of Algorithms, Springer, 2008.

[28] J. Chen, H. Fernau, I.A. Kanj, G. Xia, Parametric duality and kernelization: lower bounds and upper bounds on kernel size, SIAM J. Comput. 37 (2007) $1077-1108$.

[29] J. Chen, I.A. Kanj, G. Xia, Improved upper bounds for vertex cover, Theoret. Comput. Sci. 411 (40-42) (2010) 3736-3756.

[30] V. Chvátal, A greedy heuristic for the set-covering problem, Math. Oper. Res. 4 (3) (1979) 233-235.

[31] A.E.F. Clementi, L. Trevisan, Improved non-approximability results for minimum vertex cover with density constraints, Theoret. Comput. Sci. $225(1-2)(1999) 113-128$.

[32] E.J. Cockayne, P.J.P. Grobler, S.T. Hedetniemi, A.A. McRae, What makes an irredundant set maximal?, J. Combin. Math. Combin. Comput. 25 (1997) 213-223.

[33] E.J. Cockayne, S.T. Hedetniemi, D.J. Miller, Properties of hereditary hypergraphs and middle graphs, Can. Math. Bull. 21 (1978) $461-468$.

[34] E.J. Cockayne, C.M. Mynhardt, The sequence of upper and lower domination, independence and irredundance numbers of a graph, Discrete Math. $122(1-3)(1993) 89-102$.

[35] E.J. Cockayne, C.M. Mynhardt, Irredundance and maximum degree in graphs, Comb. Probab. Comput. 6 (2) (1997) $153-157$.

[36] B. Courcelle, The monadic second-order logic of graphs. I. Recognizable sets of finite graphs, Inform. and Comput. 85 (1990) 12-75.

[37] B. Courcelle, M. Mosbah, Monadic second-order evaluations on tree-decomposable graphs, in: G. Schmidt, R. Berghammer (Eds.), 17th International Workshop, WG'91, in: LNCS, vol. 570, Springer, 1992, pp. 13-24.

[38] F. Dehne, M. Fellows, H. Fernau, E. Prieto, F. Rosamond, Nonblocker: parameterized algorithmics for Minimum Dominating SET, in: J. Štuller, J. Wiedermann, G. Tel, J. Pokorný, M. Bielikova (Eds.), Software Seminar SOFSEM, in: LNCS, vol. 3831, Springer, 2006, pp. $237-245$.

[39] I. Dinur, D. Steurer, Analytical approach to parallel repetition, in: D.B. Shmoys (Ed.), Symposium on Theory of Computing, STOC, ACM, 2014, pp. 624-633.

[40] R.G. Downey, M.R. Fellows, Fixed parameter tractability and completeness, Congr. Numer. 87 (1992) 161-187.

[41] R.G. Downey, M.R. Fellows, Fundamentals of Parameterized Complexity, in: Texts in Computer Science, Springer, 2013.

[42] R.G. Downey, M.R. Fellows, V. Raman, The complexity of irredundant set parameterized by size, Discrete Appl. Math. 100 (2000) 155-167. 
[43] O. Favaron, Stability, domination and irredundance in a graph, J. Graph Theory 10 (1986) 429-438.

[44] U. Feige, A threshold of $\ln n$ for approximating set cover, J. ACM 45 (1998) 634-652.

[45] H. Fernau, Parameterized Algorithmics: A Graph-Theoretic Approach, Habilitationsschrift, Universität Tübingen, Germany, 2005.

[46] F.V. Fomin, D. Lokshtanov, V. Raman, S. Saurabh, Bidimensionality and EPTAS, in: D. Randall (Ed.), Proceedings of the Twenty-Second Annual ACM-SIAM Symposium on Discrete Algorithms SODA, ACM and SIAM, 2011, pp. 748-759.

[47] M.R. Garey, D.S. Johnson, The rectilinear Steiner tree problem is NP-complete, SIAM J. Appl. Math. 32 (4) (1977) 826-834.

[48] M.R. Garey, D.S. Johnson, Computers and Intractability, Freeman, New York, 1979.

[49] M.R. Garey, D.S. Johnson, L. Stockmeyer, Some simplified NP-complete graph problems, Theoret. Comput. Sci. 1 (1976) $237-267$.

[50] S. Gaspers, M. Messinger, R.J. Nowakowski, P. Prałat, Clean the graph before you draw it!, Inform. Process. Lett. 109 (10) (2009) $463-467$.

[51] A. Grady, F. Knoll, R. Laskar, D.J. Lipman, Cycle domination, independence and irredundance in graphs, arXiv:1505.02268v1, 2015.

[52] M.M. Halldórsson, Approximating the minimum maximal independence number, Inform. Process. Lett. 46 (1993) 169-172.

[53] T.W. Haynes, S.T. Hedetniemi, P.J. Slater, Fundamentals of Domination in Graphs, in: Monographs and Textbooks in Pure and Applied Mathematics, vol. 208, Marcel Dekker, 1998.

[54] M.A. Henning, P.J. Slater, Inequalities relating domination parameters in cubic graphs, Discrete Math. 158 (1-3) (1996) 87-98.

[55] Y. Iwata, A faster algorithm for dominating set analyzed by the potential method, in: D. Marx, P. Rossmanith (Eds.), Parameterized and Exact Computation - 6th International Symposium, IPEC 2011, in: LNCS, vol. 7112, Springer, 2012, pp. 41-54.

[56] V. Kann, On the Approximability of NP-complete Optimization Problems (PhD thesis), Royal Institute of Technology, Stockholm, Sweden, 1992.

[57] S. Khanna, R. Motwani, M. Sudan, U. Vazirani, On syntactic versus computational views of approximability, SIAM J. Comput. 28 (1998) $164-191$.

[58] S. Khot, O. Regev, Vertex cover might be hard to approximate to within $2-\varepsilon$, J. Comput. System Sci. 74 (2008) 335-349.

[59] J. Kratochvíl, A special planar satisfiability problem and a consequence of its NP-completeness, Discrete Appl. Math. 52 (1994) $233-252$.

[60] R. Laskar, J. Pfaff, Domination and irredundance in split graphs, Technical Report 430, Clemson University, 1983.

[61] D.F. Manlove, Minimaximal and Maximinimal Optimisation Problems: A Partial Order-based Approach (PhD thesis), University of Glasgow, Computing Science, 1998.

[62] D.F. Manlove, On the algorithmic complexity of twelve covering and independence parameters of graphs, Discrete Appl. Math. 91 (1999) $155-175$.

[63] M. Marzban, Q.-P. Gu, Computational study on a PTAS for planar dominating set problem, Algorithms 6 (1) (2013) 43-59.

[64] S. Mishra, K. Sikdar, On the hardness of approximating some NP-optimization problems related to minimum linear ordering problem, RAIRO Inform. Théor. Appl./Theor. Inf. Appl. 35 (3) (2001) 287-309.

[65] D. Moshkovitz, The projection games conjecture and the NP-hardness of $\ln n$-approximating set-cover, Theory Comput. 11 (2015) $221-235$.

[66] C.T. Nguyen, J. Shen, M. Hou, L. Sheng, W. Miller, L. Zhang, Approximating the spanning star forest problem and its application to genomic sequence alignment, SIAM J. Comput. 38 (3) (2008) 946-962.

[67] C.H. Papadimitriou, M. Yannakakis, Optimization, approximation, and complexity classes, J. Comput. System Sci. 43 (1991) $425-440$.

[68] K.B. Reid, A.A. McRae, S.M. Hedetniemi, S.T. Hedetniemi, Domination and irredundance in tournaments, Australas. J. Combin. 29 (2004) 157-172.

[69] S. Skulrattanakulchai, $\Delta$-list vertex coloring in linear time, Inform. Process. Lett. 98 (3) (2006) 101-106.

[70] P.J. Slater, Enclaveless sets and MK-systems, J. Res. Natl. Bur. Stand. 82 (3) (1977) 197-202.

[71] M. Xiao, H. Nagamochi, Exact algorithms for maximum independent set, Inform. and Comput. 255 (2017) 126-146.

[72] D. Zuckerman, Linear degree extractors and the inapproximability of max clique and chromatic number, Theory Comput. 3 (6) (2007) 103-128.

[73] V.E. Zverovich, On the differences of the independence, domination and irredundance parameters of a graph, Australas. J. Combin. 27 (2003) $175-185$.

[74] I.E. Zverovich, V.E. Zverovich, The domination parameters of cubic graphs, Graphs Combin. 21 (2) (2005) 277-288. 